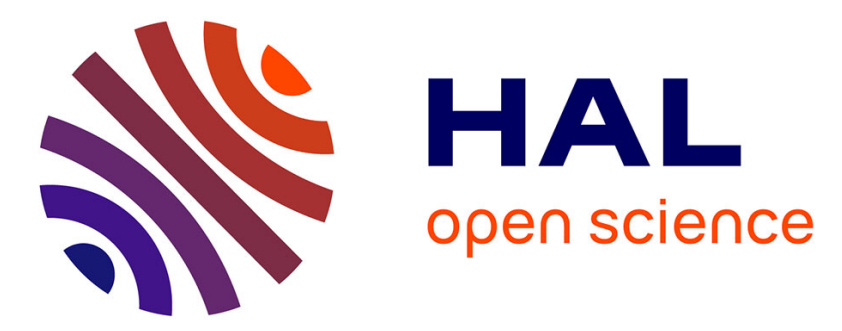

\title{
A cross layer architecture for multicast and unicast video transmission in mobile broadband networks
}

Djamal-Eddine Meddour, Abdallah Alaeddine, Toufik Ahmed, Raouf Boutaba

\section{To cite this version:}

Djamal-Eddine Meddour, Abdallah Alaeddine, Toufik Ahmed, Raouf Boutaba. A cross layer architecture for multicast and unicast video transmission in mobile broadband networks. Journal of Network and Computer Applications (JNCA), 2012, 35 (5), pp.1377-1391. 10.1016/j.jnca.2011.10.008 . hal00958083

\section{HAL Id: hal-00958083 https://hal.science/hal-00958083}

Submitted on 11 Mar 2014

HAL is a multi-disciplinary open access archive for the deposit and dissemination of scientific research documents, whether they are published or not. The documents may come from teaching and research institutions in France or abroad, or from public or private research centers.
L'archive ouverte pluridisciplinaire HAL, est destinée au dépôt et à la diffusion de documents scientifiques de niveau recherche, publiés ou non, émanant des établissements d'enseignement et de recherche français ou étrangers, des laboratoires publics ou privés. 


\title{
A Cross Layer Architecture for Multicast and Unicast transport in Mobile Broadband Network
}

\author{
Djamal-Eddine Meddour ${ }^{1}$, Alaeddine Abdallah ${ }^{1,2}$, Toufik Ahmed ${ }^{2}$ and Raouf Boutaba ${ }^{3}$ \\ ${ }^{I}$ France Telecom-Orange $R \& D$, Lannion, France \\ ${ }^{2}$ CNRS LaBRI Lab. - University of Bordeaux 1 \\ ${ }^{3}$ David R. Cheriton School of Computer Science, University of Waterloo, Ontario, Canada
}

\begin{abstract}
In this paper, we study issues related to the transport of Unicast and Multicast traffic in the mobile broadband networks. The main objective is to allow video streaming application to adapt its parameters according to 802.16 MAC layer conditions and resource availability.

For the unicast, we propose a cross layer optimizer named XLO between scalable video streaming application and IEEE 802.16 MAC layer. XLO uses the existing service flow management messages exchanged between base station (BS) and subscriber station (SS) and makes them available to the video streaming application via a specific XLO interface. We implemented the XLO in the QualNet simulator and performed extensive simulations using a personalized scalable video traffic generator, capable of streaming video with different data rates and quality level. We also introduce an enhanced admission control function at the BS that takes into account video adaptability property. The simulation results show the effectiveness of our XLO mechanism for delivering better quality of service.

For the multicast traffic, we consider that our XLO solution is inefficient since it did not handle the per user network condition. Therefore, we propose a new solution based on superposition coding and make use of scalable video coding in order to provide the best compromise between the available resource and the network condition diversity.
\end{abstract}

Keywords : - WIMAX, IEEE 802.16, Video Streaming, Multicast, Unicast, Cross Layer Optimization.

\section{Introduction}

With the prevalence of the Internet today and the diversity of technologies supporting multimedia services, wireless technologies continue to progress in order to provide maximum bandwidth and coverage with the best quality of service and experience for the users.

IEEE 802.16 [1][2], is a broadband, high data rate wireless technology. WIMAX technology is intended to support multimedia applications such as real-time video streaming and VoIP. In order to perform efficiently, real time applications require strict resource reservation, namely bandwidth guarantee and bound on the maximum transmission delay, jitter and loss rate. However, the availability of the network resources is subject to network conditions notably at the physical (PHY) and Medium Access Control (MAC) layers. To respond to the lack of resources, video streaming applications should ideally, adapt their data rate according to changes in network conditions observed at the PHY and MAC layers. This process is called awareness and is used in many crosslayer optimisation mechanisms.

Cross-layer design is an ongoing research topic aiming at increasing the Quality of Service/Experience (QoS/QoE) by performing coordinated actions across different network layers and, thus, violating the protocol layered and isolation model.

In this paper, we designed two separate paradigms to handle both unicast and multicast traffic. For the unicast, we proposed a cross layer optimizer, named XLO which facilitates the information exchange between streaming applications and IEEE 802.16 MAC layer to perform rapid and accurate video quality adaptations according to physical network conditions. Then, we developed a video quality aware admission control function at the BS aiming at guaranteeing the expected QoS. The objective is to exploit the scalability property of video stream and adapt the video quality with respect to the available resource. For the multicast, we proposed a new solution which optimises the delivery of scalable video stream based on superposition coding schema able to provide the best compromise between resources availably and network condition diversity. 
The rest of this paper is organized as follows. Section 2 presents the related work, then we define the system architecture and the targeted scenario in section 3 . The performance evaluation of the solution is presented in section 4 . The conclusion is provided in section 5 .

\section{Related Work}

\subsection{Cross Layer optimization}

Most previous works on cross layer optimizations in WIMAX networks focus on PHY and MAC layer interactions and do not explicitly consider the application layer (APP) performance. Authors of [3] and [4] propose a Cross layer framework to integrate layer 3 and layer 2 QoS. They introduce a fragment control mechanism which enqueue all fragments coming from different IP packets in the same buffer. They also propose a remapping scheme for better buffer utilization allowing a low frame dropping ratio. In [5], the authors propose a cross layer optimizer between MAC and PHY layer. Their solution collects parameters like channel condition information, bandwidth requests and queue length from both layers and returns optimized parameters back to the two layers.

In [6] and [7], the application layer is included in the cross layer mechanism along with the MAC and PHY layers. Optimization is performed at the BS which delivers video traffic to SS. The authors of [6] utilize information provided by the PHY, MAC and APP layers to improve system performance. The main idea is to adapt and adjust modulation in MAC layer and data rate of the video streaming application depending on channel condition. They introduce new management messages to inform SS about video data rate. In [7], the authors conduct a performance evaluation study of a cross layer approach for multiuser H.264 video transmission in wireless networks. Three steps are defined: abstraction of parameters from the APP and Radio Link layers; selection of optimized parameters and their distribution to corresponding layers. A cross layer algorithm is applied in the beginning of each frame for all users simultaneously which adds a significant overhead.

Our work considers cross layer optimisation between Application and MAC layers at the server-side in the SS and not at the BS as in [6] and [7]. Moreover, in our approach, we do not add any new management messages but rather exploit existing MAC management messages. Finally, to reduce computation overhead, our optimization is performed only at the beginning of a video streaming session or during the life-time of a session if any changes are detected in the MAC layer conditions.

In the next subsection, we discuss QoS support in the IEEE802.16 MAC layer.

\subsection{QoS design \& service flow management in IEEE 802.16}

Each packet passing through the IEEE 802.16 MAC interface belongs to a service flow (SF) identified by a connection identifier (CID). A SF is a unidirectional flow of packets providing a particular QoS assurance in terms of a set of QoS parameters such as latency, jitter and throughput. A SF may be created, changed or deleted using a MAC management message: DSA, DSC and DSD.

The remaining of this subsection describes these messages which are essential for our cross-layer optimizer.

\subsubsection{Dynamic service flow add message (DSA)}

Creation of a SF is initiated by either SS or BS. If SS is the initiator, the message contains reference to the concerned SF, QoS parameter sets or service class name. The BS responds by DSA_RSP message indicating the acceptance or rejection of the request. The rejection message may contain extra information such as unsupported parameters or wrong values. When the BS is the initiator, the DSA-REQ message contains SF identifier (SFID), CID and active or admitted QoS parameters. The response of the SS is the same as in the first case.

\subsubsection{Dynamic service flow change message (DSC)}

After its creation, a SF can be modified via the DSC-REQ message. The Modification includes admitted and active QoS parameters. Changes are done when both admitted and active parameters are replaced. In fact, if only admitted parameters are included in the message, active parameters will be set to null and SF will be deactivated. When both parameters are inserted in the message, admitted sets are verified first by the admission control mechanism and then the activated sets to ensure that it is a subset. If successful, changes take place in the SF; otherwise, the SF become unchanged and continues performing with existing parameters.

\subsubsection{Dynamic service flow delete message (DSD)}

A SF is deleted from BS or SS through DSD_REQ message. Moreover, a SF may be deleted implicitly when errors or mistakes occur. The SF management messages will be used in our approach to help XLO fix optimized 
video streaming parameters and consequently provide better delivered quality of service. Details of our approach are described in the next section.

\subsection{Supporting heterogeneous clients in WiMAX}

The transmission of an IP video stream would be largely facilitated if the clients share the same radio conditions. However, due to the inherent characteristics of the radio medium in real world scenario, the mobile clients who are attached to a WiMAX BS have heterogeneous capabilities. Figure 1. shows clearly this phenomenon with different users supporting 64QAM, 16QAM, and QPSK coding.

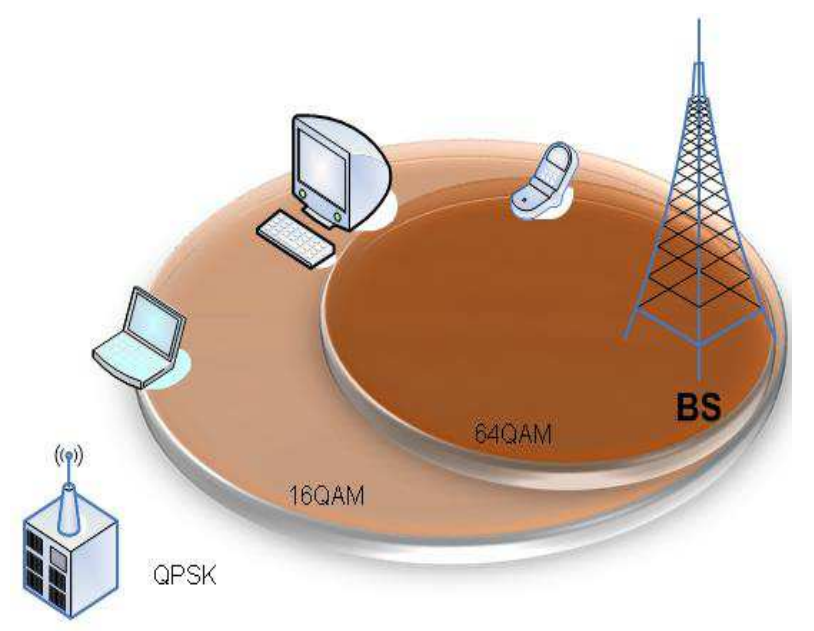

Figure 1. Low increased to High quality video data rate during transmission

Indeed, the closer the SSs are to the BS, the higher the bandwidth they have and the better is the quality of experience for the user. In the other hand, the SSs which are not closer enough to the BS will not obtain the needed resources to meet the video streaming requirements. To overcome this issue, adaptable policies could be established at the video server-side to manage the network resources effectively and hence to provide the appropriate QoE. In this case, the multicast approach cannot be applied because of the heterogeneity of user profiles present in the cell. Below we detail the limitations of IP multicast for different cases:

- Case 1: The transmission of a video stream with the highest quality to satisfy the most favored client, with enough resources. In this case, all other users who do not meet the criteria of the video stream in terms of resources will fail to receive the video stream with a correct quality (due to signal attenuation).

- Case 2: The Transmission of a video stream with the lowest quality to satisfy all the users. In this scenario, users with higher bandwidth are jeopardized and will receive a lower quality video.

- Case 3: Transmission of a video stream with an acceptable for the majority of the users. This increases the number of the satisfied user with the perceived video quality but does not solve all the issues. Some users with good radio condition will not receive the video at the best possible quality; others will not receive any video.

- Case 4: Transmission of a video stream several times with a different degree of quality. The video server may, depending on the profiles of its users run multiple video streams with different quality (minimum, average and maximum). This solution can satisfy a greater number of users. If the video profiles of different users are very close, the number of video streams to be transmitted is reduced. In contrast, if the diversity of profiles is important, the number of video streams cannot solve the problem. Despite the simplicity, this solution has the biggest disadvantage to increase the bandwidth required and the resources necessary to transport different qualities of the same video stream.

As we can see clearly, none of the presented solutions deal correctly with the problem of diversity in terms of network resources. Depending on the cases, some users will experience a degradation of their quality of experience QoE, the network resources will not be managed effectively, or that some users will be ineligible to serve. Ideally, the perceived video quality by each user should correspond to its capabilities and the available resources.

The required solution needs to takes into account all these constraints. We kept our focus on the recent developments of video coding supporting the heterogeneity of user profiles and network environments. Towards a better support of users, we investigated the hierarchical coding of SVC (Scalable Video Coding) for multicast 
services support. Indeed, the SVC techniques appear to be the most appropriate video coding solution that meets the requirements mentioned above.

In the next paragraph, we detail first, some examples of multicast video solution based on the hierarchical video coding. Then we present our solution and we detail our architecture and its performance evaluation.

\subsection{Hierarchical coding for multicast support}

There are several studies devoted to the use of hierarchical coding technique for multicast video transmission. We describe below two main proposals.

SVSoA approach is defined in [10]. The authors present a solution for streaming video using hierarchical multicast IP. By using the protocol ALC (Asynchronous Layered Coding) [11], SVSoA guaranteed the scalability vis-à-vis the heterogeneity of the users and the reliability. Though, the ALC protocol is considered just as a reliable multicast transport protocol and is not designed primarily for real time transmissions.

The authors combine hierarchical video coder with the ALC protocol. Indeed, the video server split the video into segments of equal duration. Each segment is composed of several blocks so that each block represents the base video layer or an enhancement layer. The transmission of each block is performed independently in an ALC session. Thus, each layer video is transmitted in a different multicast group. Upon receipt, each client begins by retrieving data from the first ALC session which contains the base video layer. Then, before the end of the segment duration, the client switches to the session that corresponds to the ALC video layer enhancement. This process is repeated for all enhancement layers. In this paper, the authors considered a session for the base layer and another for a single enhancement layer. SVSoA uses a fixed duration segment of 60 secs, and as the reception of the layers is sequential, the playback can begin before the full acquisition of the segment. Thus, a higher latency is observed compared to a conventional architecture where the segment size is the size of an image (1/25 s). According to the authors, SVSoA solution is valid for all hierarchical video coding schemes.

The drawback of this solution lies in the increasing number of active ALC sessions. Indeed, the server must send all the video layers and thus create a large number of ALC sessions even if the user will only receive the base layer video. The second issue not addressed in this approach concerns the heterogeneity of receivers. Finally, we must ensure that the duration of the video segment will allow the user to receive the maximum enhancement layers in addition to the base layer.

The authors of [12][13] propose a Cross-Layer Architecture for IPTV video transmission in a WiMAX network. Indeed, the proposed solution aims at finding certain compromise between an application such as hierarchical video coding MDC and the radio channel diversity of the users. Then, Cross-Layer approach is adopted. This solution takes into consideration the features of the MDC video applications and offers a modulation scheme for optimal resource allocation to maximize video quality even under the worst radio conditions.

In [14], authors propose a new modulation scheme for WiMAX networks, allowing a better multicast transmission. Each multicast stream is transported differently depending on the required quality. The basic video layers will be streamed using a BPSK modulation scheme, for the enhancement layers a better modulation such as 16QAM will be used. Thus, a receiver can get the basic video quality by decoding partially multicast packets flow modulated BPSK channel when the radio condition is deteriorating, or get the best video quality from all streams (both BPSK and 16QAM channel).

A modification to the MDC coding is introduced by the authors by applying a proper protection scheme to the MDC base layer. This modification is essential to use the proposed coding technique. Indeed, while protecting the packet modulated with BPSK is not necessary, for 64QAM modulation, many losses can occur and extra protection will provide coding reliability of the transmission.

We have seen in this section some solutions to multicast video transmission, taking into account the hierarchical coding of video streams and the diversity of users and the radio channel access. These proposals have allowed us to define our multicast architecture using the SVC coding. We present this architecture in detail in the next section and the various technical components that comprise it.

\section{Target Scenarios \& System Architecture}

\subsection{Unicast Services Support}

We focus on scenarios for providing unicast services where to users. In this scenario, different SSs share their video in real time. The appropriate use cases of our approach are video conferencing, video surveillance and $\mathrm{P} 2 \mathrm{P}$ video delivery where unicast stream is appropriate. The main idea of our work consists of optimizing video streaming application initiated by SSs in Uplink channel.

Figure 2. shows a video streaming traffic between 2 SSs associated with different BSs (BS1 and BS2). 


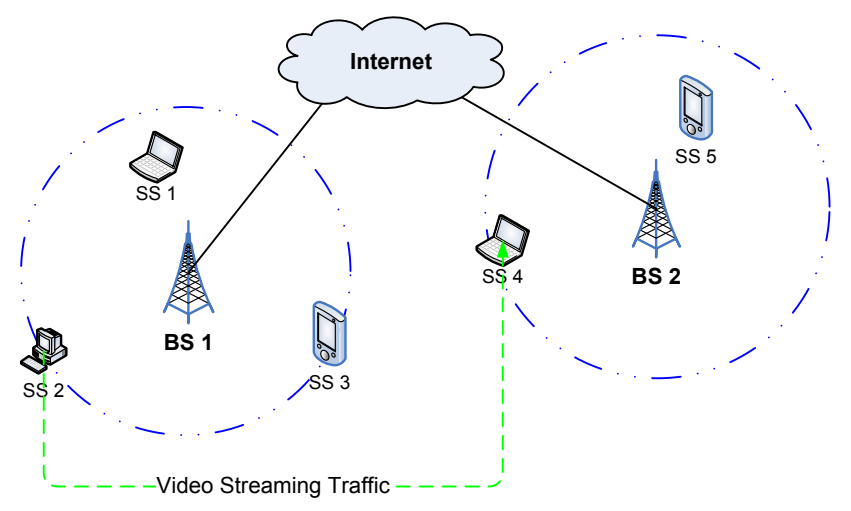

Figure 2. System Architecture

Our approach consists of an adaptation mechanism at the video streaming application jointly with cross layer mechanism between MAC and Application layers. The adaptation occurs at the server-side, i.e., the owner of the video content. The proposed design for the protocol stack interactions is illustrated in Figure 3.

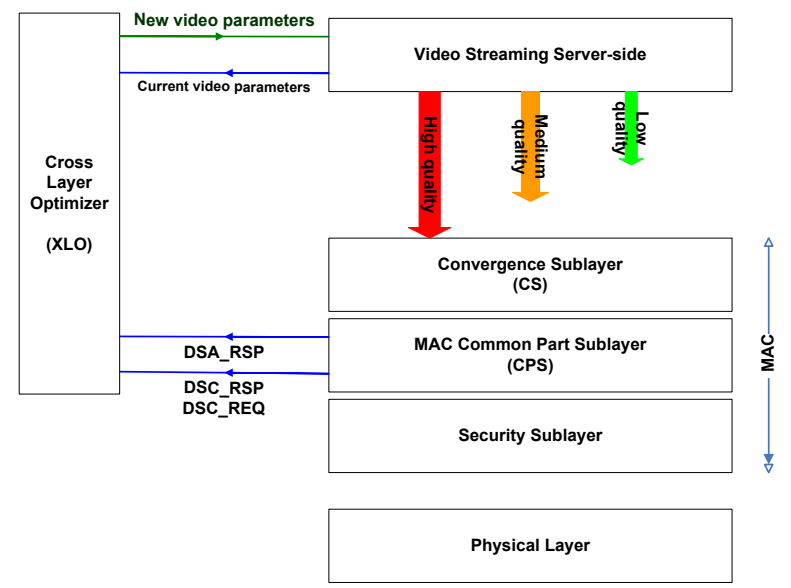

Figure 3. Cross layer optimizer between MAC and application layer

In this figure, we can see the reference model essentially for MAC and PHY layers as defined in IEEE 802.16d standard [1] and the XLO. The XLO makes available the SF management messages (DSA, DSC, and DSD) to video streaming server-side, which in turn, makes the video parameters available to XLO later from the beginning of the streaming session.

The CPS (Common Part Sub layer) provides the core MAC functionality of system access, bandwidth allocation and connection establishment. In particular, CPS is responsible for SF management. Thus, the XLO interacts with CPS sub-layer and video streaming server-side application layer.

\subsubsection{Cross Layer Optimization Algorithm}

The devised cross layer optimization algorithm consists of 3 steps. First, we collect information from CPS sublayer. Second, this information is analysed and a decision is made at the XLO. Finally, we apply the adaptations by enforcing the new video parameters at the video streaming server-side.

\subsubsection{Information collection}

As mentioned in section 2.2, SFs are managed (added, changed and deleted) via DSA, DSC and DSD messages respectively. Many messages are exchanged between SS and BS, and we are interested especially in DSA_RSP, DSC_REQ and DSC_RSP messages. The main idea consists in using existing management messages rather than adding new ones. Then, since our approach is implemented in the video streaming server-side, a copy of all SF management messages initiated by the transmitter station or received from the BS will be collected by XLO. 


\subsubsection{Adaptation and modification}

The collected SF management messages are first analysed. These messages contain a positive response if the request is accepted and a negative response if the request is not totally accepted or rejected.

If the request is not accepted, it indicates the unavailability of the needed resources for the video stream. Hence, the video streaming application has to adjust its behaviour in order to get a positive response in the next attempt. For this end, we modify the streaming parameters so as to adjust the video data rate throughput. This procedure may be repeated until the video stream is accepted or we reach the minimum video quality supported with a reject response. The selection of the minimum and maximum video quality level usually negotiated using existing signalling protocols such as SIP, RTSP or H323 and can be part of the service level agreement. This is however out of the scope of this paper.

Once the request is accepted, the information and the time of acceptance are stored. Then, if the video stream performs correctly for a certain period, the XLO will increase the video data rate assuming that more resources are available. If the request is rejected, the video stream will continue using its current parameters. If accepted, the same process is repeated until we reach the maximum video quality. The algorithm describing this process is detailed below.

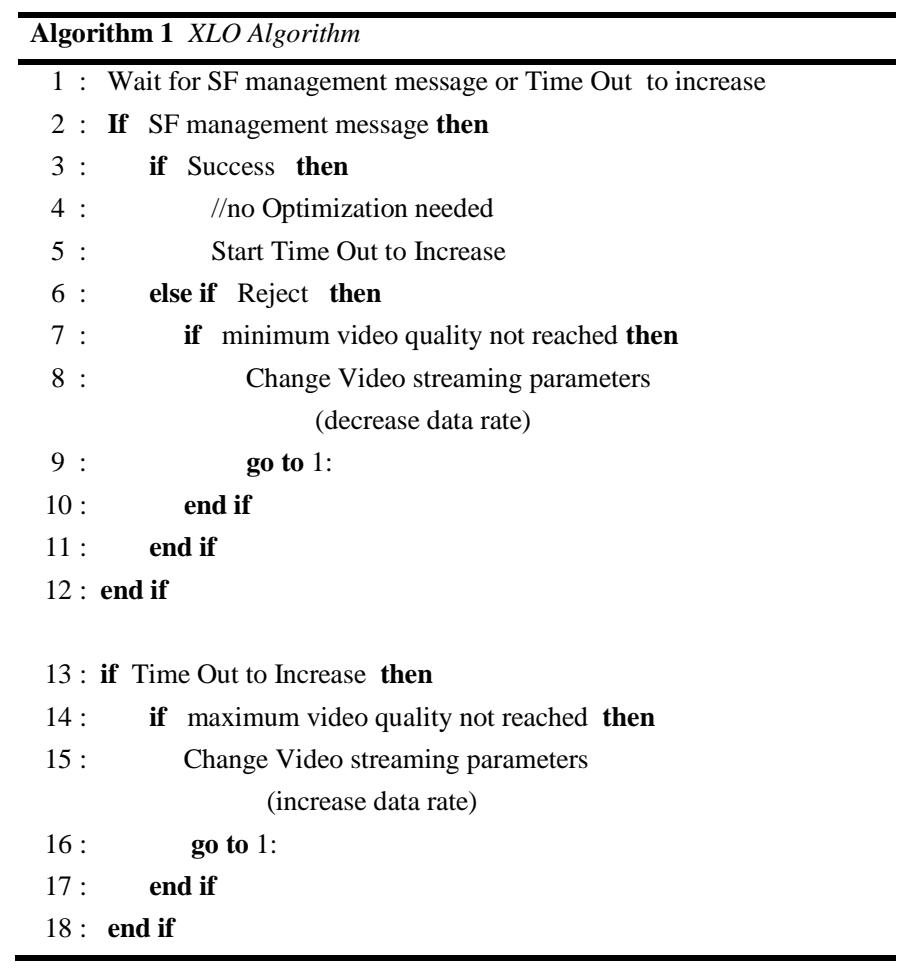

The next subsection presents the sequence diagrams that provide more details about the cross layer optimization operations and the exchanged messages between the entities involved in the optimization process.

\subsection{Illustration of the approach}

When a new video is requested, the XLO allows us to identify the suitable video parameters that guarantee the acceptance of the corresponding SF at the MAC layer. During the video streaming, the changes occurring in the MAC layer conditions and consequently in the video throughput may force the service to be stopped or rejected. In the absence of our XLO, the video stream will abort if the SF parameters do not meet its QoS constraints. The XLO allows us to adapt video streaming data rate according to the fluctuation of the network conditions and hence avoids the SF rejection. Furthermore, our approach can be applied at the service request / invocation as well as during the life-time of the session or at service delivery.

In the following, we will illustrate our approach through some examples. We use the topology presented in Figure 2., where in the SS2 station we implement a video streaming server with XLO capability.

\subsubsection{SS initiated DSA request message}

When the MAC layer in SS2 receives a video, it initiates a DSA_REQ message with the desired QoS parameters and sends it to the BS1, which checks the integrity of the message and responds by DSX_RVD (DSA or DSC 
received) message. Then, depending on the acceptance or rejection of the QoS parameters, BS1 sends DSA_RSP message with a success or reject code.

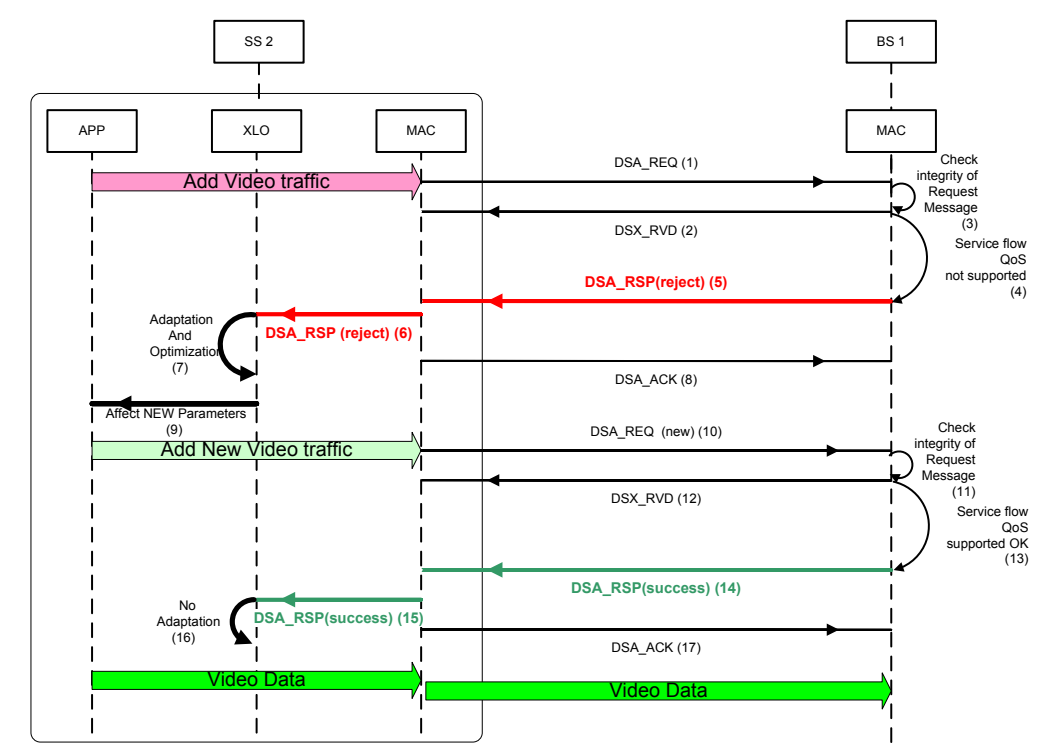

Figure 4. SS initiated DSA Request Message

In Figure 4. , we present the sequence diagram where a new SF is rejected in the first attempt and accepted in the second attempt, thanks to XLO. In fact, once the SS receives a DSA_RSP reject message (step 5), it forwards it to XLO (step 6). The latter intercepts the message and commands the video streaming application to adapt its QoS parameters by reducing its video data rate (step 7 and 8). Then, a new video add request is initiated. The same procedure between BS1 and SS2 is performed. The request is accepted and the video streaming started.

After the acceptance of a new video flow, the channel conditions and availability of resources might vary. In this case, BS or SS should send a DSC request message in order to change service flow QoS parameters.

\subsubsection{BS initiated DSC request message}

The sequence diagram depicted in Figure 5. shows a BS initiating a DSC request message (step 2). In fact, a BS should send a DSC request message once it is unable to meet the new SF QoS constraints. This might happen in two cases, when the required resources at the BS side are unavailable, and when the QoS constraints cannot be satisfied by the link between BS1 and the receiver station SS4, BS1 sends a DSC request message to SS2 in order to adapt the SF parameters accordingly. Then, SS2 receives the request and changes SF parameters consequently (step 3). 


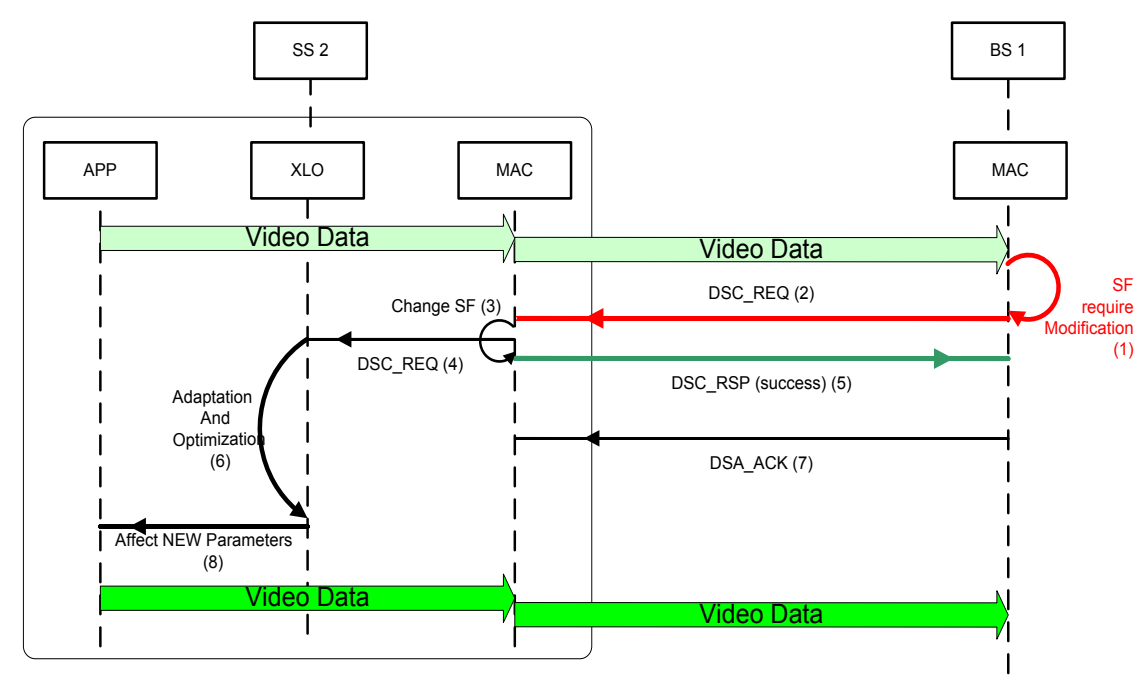

Figure 5. BS initiated DSC Request Message

\subsubsection{SS initiated DSC request message}

An SS must initiate a DSC_REQ message if it detects changes in the channel conditions. In this case, it requests BS1 to change corresponding SF QoS parameters by increasing or decreasing the related video data rate. Once the BS1 receives DSC_REQ message, it replies by DSX_RVD message if the request message is valid (step 4 in Figure 6. ). Then, it checks if the new SF parameters can be supported and sends DSC_RSP message to SS1 with a reject or success response.

If the request is rejected, SS1 will continue with its current parameters. If the request is accepted as mentioned in step 6, it sets up new SF parameters. Therefore, the XLO avoids the rejection of new video SF by adapting the video streaming parameters accordingly (step 8 and 10).

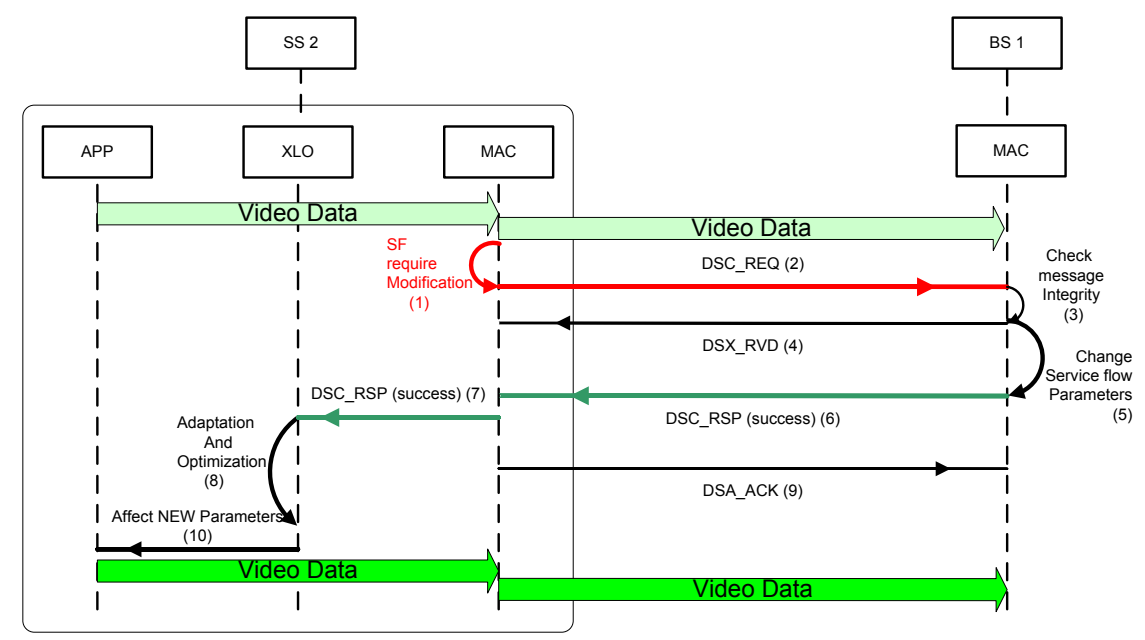

Figure 6. SS initiated DSC Request Message 


\subsection{Multicast services support}

\subsubsection{Mulicast group creation with SVC coding}

Unlike many coding schemes that generate a single video stream with a single layer, the SVC generates multiple streams to multiple hierarchical levels, the basic layer (BL: "Base layer") and one or more enhancement layers (EL: "Enhancement layer"). The base layer is sufficient for decoding, but the decoding of enhancement layers require decoding of the base layer and all depending enhancement layers. Hierarchical levels can be constructed in three dimensions temporal, quality (SNR) and special as depicted in Erreur! Source du renvoi introuvable.Figure 7 .

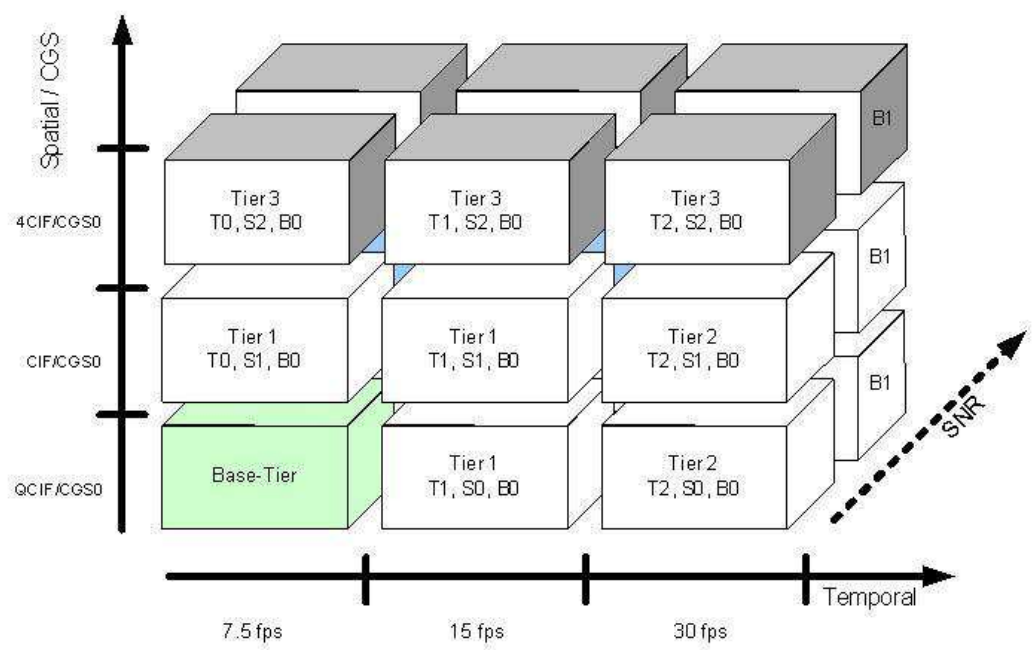

Figure 7. SVC hierarchical level

- The time dimension refers to the number of frames per second (frame rate). The base layer consists of I and $\mathrm{P}$ frames and the enhancement layers are composed of B frames inserted between images I and P.

- The spatial dimension refers to different image sizes. The upper layers provide a larger image.

- The quality dimension SNR is the image quality. The upper layers allow a finer quality of the image with more precision.

With hierarchical coding each user will receive at least the basic layer, and then, depending on the available bandwidth, it will receive a number of enhancement layers. With this distribution, we guarantee that each user will have the best possible video quality.

To handle the users heterogeneity correctly, we investigated a cross-layer architecture that combines the features of the SVC hierarchy, the IP multicast transmission and physical layer characteristics of the WiMAX network. Transmitting a multicast SVC stream requires prior knowledge of available resources in the network to optimize its delivery. However, this knowledge is highly dependent on the type of traffic sent and the number of users in the cell. Several combinations of transmission may be possible:

- Associate a multicast group for each video layer, one for the base layer and each enhancement layer. In this case, the number of multicast sessions is proportional to the number of layers. A multicast session is defined by a multicast address of class D.

- Associate a multicast group for several video layers. We may associate a multicast group to the base layer alone can group together a number of enhancement layers in the same multicast group. This solution is interesting and presents several alternative placements. Indeed, the choice of video layers can be grouped together according to several criteria.

Therefore, we opted for the generation of multicast groups according to the temporal axis which corresponds to the number of frames per second. We note that other possibilities remain compatible with our approach. Each multicast stream contains the highest quality spatial and SNR. This assumption is made mainly to simplify the simulation study and does not affect the design paradigm of our solution. An example of decomposition into several multicast groups is depicted in Figure 8. . 


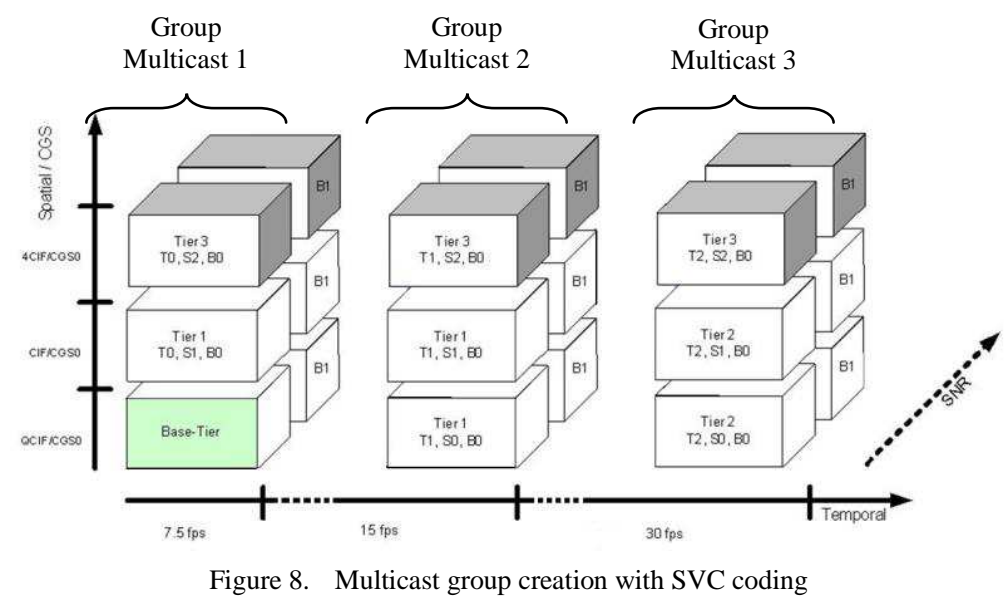

As a direct result of the SVC video encoder, the multicast groups created are complementary. In fact, according to the available bandwidth, a client should join the first multicast group, which represents or includes basic video layer to decode the enhancement layers. Then, according to its available bandwidth, the SS joins the second multicast group, and so on. To receive the highest quality video, a client should receive the data from all multicast groups. Thus, there is a direct correlation between the weight of multicast groups and the importance of video layers they contain.

So far, we have explained how the application uses the SVC coding scheme, how to create the multicast groups according to the video priority. In the following, we describe some aspects related to the MAC and physical layers used in our solution. First, we explain the normal functioning of such a multicast architecture within a WIMAX network. Then, we describe the proposed improvements, in particular at the radio level, to maximize the use of radio resources and to maximize users video quality.

\subsubsection{Mulicast transmission for the WiMax networks}

To transmit a data stream, a request to add service flow should be sent to the base station even if it is the BS that must initiate the traffic. Then, the function of admission control occurs. It is important to note that this procedure is valid for both unicast and multicast flows. More details are given bellow.

\subsubsection{Admission control in WiMax networks}

We recall that the admission control is a function that depends on the manufacturer, the IEEE 802.16 standard does not specify the scheduling algorithm, it only defines the various tools such as the signalling messages and the operation base.

Indeed, the calculation of available resources depends on the size of the frame to the PHY level, the frequency band and modulation and coding used. Thus, after fixing all these parameters, it would be feasible to calculate the number of slots, a WIMAX physical station features. Recall that a physical slot allocation consists of a rectangular symbol OFDM time and frequency.

Suppose that the BS wants to send a data stream to one of the SSs of the cell, it begins by calculating the number of slots needed for physical flows (after consultation with all PHY parameters corresponding to both the BS and SS question). Then, the BS checks the availability of that number of slot and accepts or denies access to this stream.

Obviously, the SS will be informed of this decision via signalling messages and management of service flows. In particular, each SS receives the resource allocations in the MAP field at the beginning of each frame just after the preamble.

\subsubsection{The DL_MAP message}

At the beginning of each frame, the BS includes messages and DL_MAP UL_MAP that are broadcasted to all SSs in the cell. The DL_MAP contains the description of resource allocations for each SS for each data stream. The DL_MAP allows the SS to locate the burst of data that is dedicated. The burst is a part of the frame identified by a time range and frequency. Each burst is described in the form of a DL_MAP DL_MAP_IE. This provides the exact location within the frame.

According to IEEE 802.16, the DL_MAP field did not contain any information to describe the coding and modulation scheme used. Indeed, following the negotiations between the BS and SS, the modulation scheme and coding is fixed. It can be updated when changing radio conditions, the BS informs of changes through the UCD and DCD fields which are sent periodically by the BS at the beginning of the frame. The value of the 
modulation and coding is used for all flows sent to the same SS. Thus, an SS may use two different modulations in the same frame for example.

Since a SS which can receive a 64QAM encoded data, it is quite capable of receiving data with more robust modulations such as BPSK or 16QAM, especially because UCD and DCD fields, and DL_MAP UL_MAP are all encoded BPSK so that all the SSs are lossless. The same is observed for the containment area dedicated to bandwidth demand by contention between all SSs. Subsequently, it is quite possible to tell the SS receive data with different modulations that do not exceed the maximum negotiated with the BS.

Information containing the modulation scheme and coding used for any burst will be included in the corresponding DLMAP_IE. This detail is necessary for the implementation and comparison of two approaches that we describe in the paragraph below.

\subsubsection{Proposed architecture for supporting multicast services using SVC and superposition coding scheme}

In this section we study the various possible topologies in WiMAX networks to support multicast services. Indeed, there are several possible scenarios.

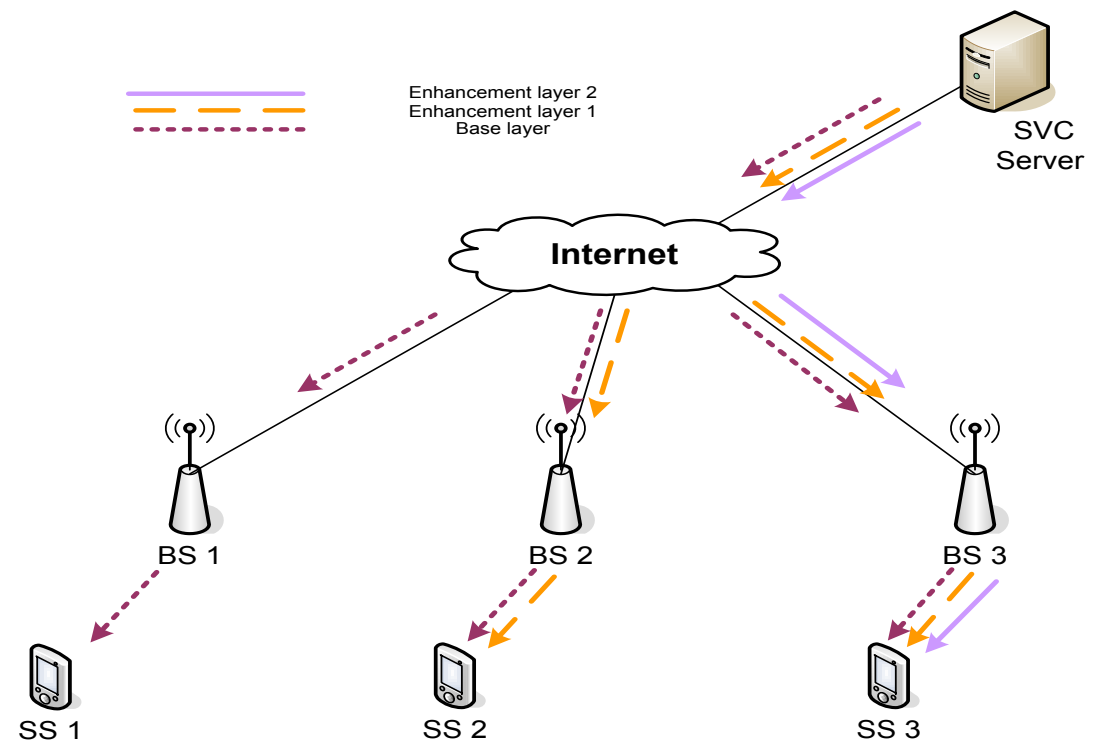

Figure 9. Multi-Cell Architecture (Multi-BS)

Initially, we consider the topology shown in Erreur ! Source du renvoi introuvable.Figure 9. . We have a server with a video encoder capable of transmitting video SVC flow in the form of several multicast sessions as it was presented in the previous paragraph. We assume that the flow is an SVC stream which consists of a base layer and two layers of improvements (layers are seen as groups providing each an enhancement of the final quality level). The multicast groups for the three quality level are represented by small arrows (see Erreur ! Source du renvoi introuvable.Figure 9. Erreur ! Source du renvoi introuvable.). We also assume that there are three WIMAX cells connected to the wired network. An SS of each cell, connects to the server and requests the video stream in real time. According to their respective bandwidths from start to finish, each SS will be a member of one or more multicast groups. The SS1, for example, it will be able to receive only the multicast session which is the base layer video stream of the SVC. This may be due to the unavailability of radio resources within its cell. The SS3, for cons, will be able to receive all three layers of video and will result in the highest available video quality.

In this case, we note that the multicast streams are transported from the video server to the base stations of each cell. Note that the third stream which corresponds to the second enhancement layer has not been sent to the BS1 and the BS2, while the first stream (Base Layer) was sent to all the BSs. Thus we conclude that the decomposition of the SVC flows into different multicast stream is partially sufficient, in particular for this kind of topology. For SS belonging to different cells, they will receive only the multicast stream which corresponds to its capabilities and the available resource. This also remains valid if different SSs belong to the same cell, provided they have the same radio characteristics. In this case, no additional resources are required. 
We present now our approach within a single cell. The Erreur ! Source du renvoi introuvable. depicts the same video server SVC and three SSs with different radio conditions belonging to the same BS.

We assume that the three SSs are at different distances from the BS so that the BS assigns to each SS a modulation scheme and different encoding. For example: 64QAM SS1, SS2 and SS3 in 16QAM QPSK.

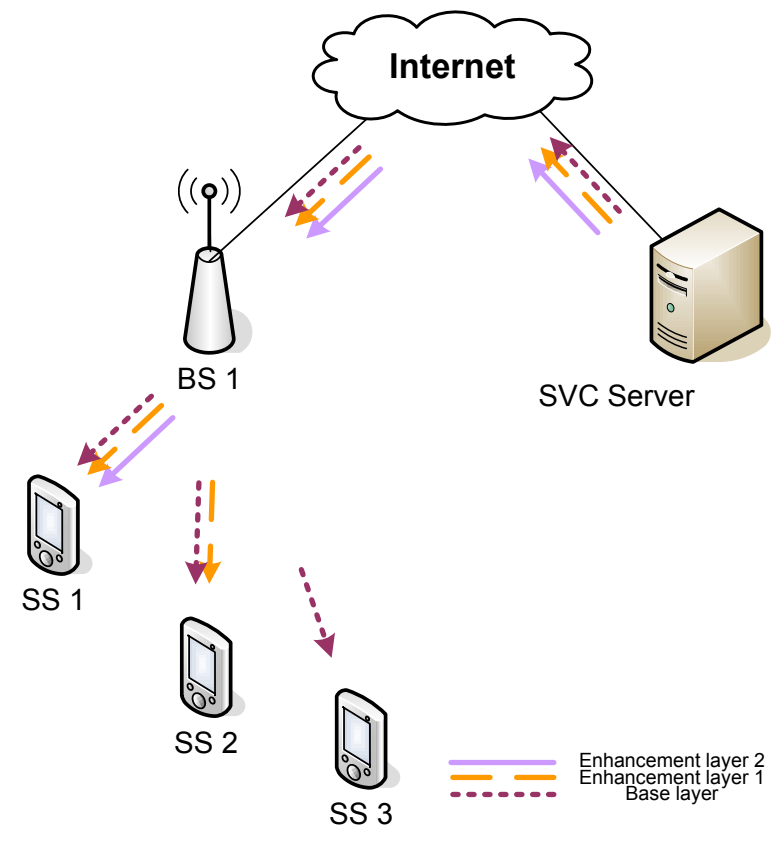

Figure 10. Single cell Architecture

\subsubsection{Simple modulation scheme}

For each SS that receives the best video quality, it must adhere to the three multicast groups and retrieve all the video layers. Once the multicast stream arriving at the BS, a request to create a new flow of service for each group and each SS is generated. The BS must duplicate each multicast stream since SSs have different modulation schemes. Thus the video base layer is transmitted three times within the same cell, once in QPSK, 16QAM and once again in 64QAM. The same is done for the other two video layers. The BS will allocate the necessary resources for each new service flow. Obviously, in case of radio resources lacking, the BS will prioritize the transmission of the base layer and enhancement layers. Similarly, the most favoured SSs, closest to the BS in general, will be treated first. The scheduling mechanism of the BS must take into account these two criterions.

Apart its simplicity, this method has several disadvantages:

- Resource management is far from efficient. Indeed, the same data are encoded several times, this redundancy disservice to the very principle of multicast transmission. This is a simultaneous transmission of the same video with different modulations and possibly with different qualities.

- The BS has a limited number of physical slots to allocate. With these, the BS cannot satisfy all requests. Each SS must reduce the quality of the video each time there is a new SS or not compatible with characteristics different radios.

- The SSs with better reception quality are those closest to the BS. The coding and modulation scheme used is less resource demanding, while the later SSs may waive the video stream due to a lack of resources.

- The SSs, supporting a less robust modulation, are quite capable of decoding more robust modulation. Thus, these SSs can decode the same video stream as many times there is modulation scheme used.

To overcome these limitations, we propose as a first step, a new system of allocating resources to distribute the multicast stream for different modulations.

\subsubsection{Multiple modulations scheme}

The idea is to benefit from the fact that SS is capable of operating with a less robust modulation, can also work with a more robust modulation. Indeed, by taking the topology described in Erreur ! Source du renvoi introuvable., the multiple modulations mode is performed as follow: 
- The base layer is modulated in QPSK, the burst of data containing the first service flow is visible for all the SSs without exception. Thus, the minimal video quality is already earned for all the SS in the field of coverage of the BS.

- The first enhancement layer is coded 16QAM, the corresponding data burst will be visible only by SS1 and SS2. (i.e., only stations that can decode this modulation)

- The second enhancement layer is coded 64QAM, the corresponding data burst will be visible only by SS1.

In this example, we note that each SS receives a different video quality. The video segments are encoded with modulations in descending order of strength. More redundancy is omitted and the resources are used much less. The resources released can be used to improve video quality for all SSs. For example, the coding of the base layer and first enhancement layer may be made in QPSK and the opportunity to SS3 receives enhanced video quality.

Specifically, we modify the function of admission control at the BS to determine the optimal distribution between multicast groups and different modulations used in the cell. This is to maximize the video quality received by each SS based on the available resources in the radio channel. The algorithm for allocating resources to different video stream is described as follows:

- Perform the list of SSs wishing to receive the video stream.

- Establish respectively the modulation scheme of SSs in descending order of strength.

- List the flows of services related to multicast groups according to the size of the video layer that transports.

- Calculate the number of slots required for each physical flow of service with each modulation.

- To ensure that maximum SSs receiving the video stream, the BS tends to affect the modulation as robust as possible to all streams of service within existing resources.

- If resources are exhausted and there is still another layer to improve video that is not yet been transmitted, the BS needs to change the modulation of one or more previous layers to a less robust modulation to free up resources for new flows. The new stream will be encoded with the least robust modulation already used. Note that this change did not affect IP multicast

In the best case, all layers of video are encoded using the most robust modulation and thus all SSs receive the same video with better quality. The worst case is that the resources available do not allow the transmission of video streams with the least robust modulation and thus only a small number of SSs will have access to the video.

Multi-mode modulation benefits from the diversity of patterns of modulation of SSs, part of the same cell, and the diversity of groups representing the multicast video layers SVC. The perfect combination between these two parameters, taking the available resources into account, allows the BS to distribute video streams to different SSs in a fair and optimum.

In the next section we introduce another technique which saves considerable resources. Unlike the multi-mode modulation, this technique allows each SS to use a single modulation scheme pre-negotiated between the SS and the BS.

\subsubsection{Superposition coding scheme}

According to IEEE 802.16, the result of scheduling at the BS is to allocate resources for each service flow for each SS. The allocation information is transmitted via the message DL_MAP to all SSs. Each SS has the location of the burst data in the frame. The burst of data allocated to it is encoded with the most appropriate modulation according to its terms radios. Since it is designated to other SS,.The rest of the burst can be encoded with a different modulation.

Let consider the topology defined in Figure 10., with the SVC server and SSs with different modulations. Usually, multiple burst data will be created and each with a different modulation by SS destination. Each SS receives its data in the burst that is intended, and remains idle during other burst carrying the other streaming video / data to other SSs.

If multiple SSs with different modulations could use the same burst of data, a considerable gain of radio resources is achieved. Indeed, if the BS reaches coding of OFDM symbols with two or more modulations at the same time, each SS decodes these symbols with OFDM modulation and recovers its own data. This technique is called superposition coding [13][14] and was designed to improve the user capacity in wireless networks.

An example is illustrated in Figure 11. , the nodes are indexed in ascending order according to their distance from the BS. As shown, when the BS transmits a signal to M3 with a certain SNR level, the SNR experienced by both M1 and M2 is much larger than their expected level of SNR or sufficient. Similarly, when the BS 
transmits a signal to M2, M1 receives extra power over its SNR level. This implies that M1 has a sufficient SNR to decode messages intended for both M2 and M3, and M2 has a sufficient SNR to decode messages intended for M3. Thus, information intended for M1 can be included in the transmission or M2 to M3 by using the superposition coding. Similarly, the information for M2 can be included in the transmission to M3.

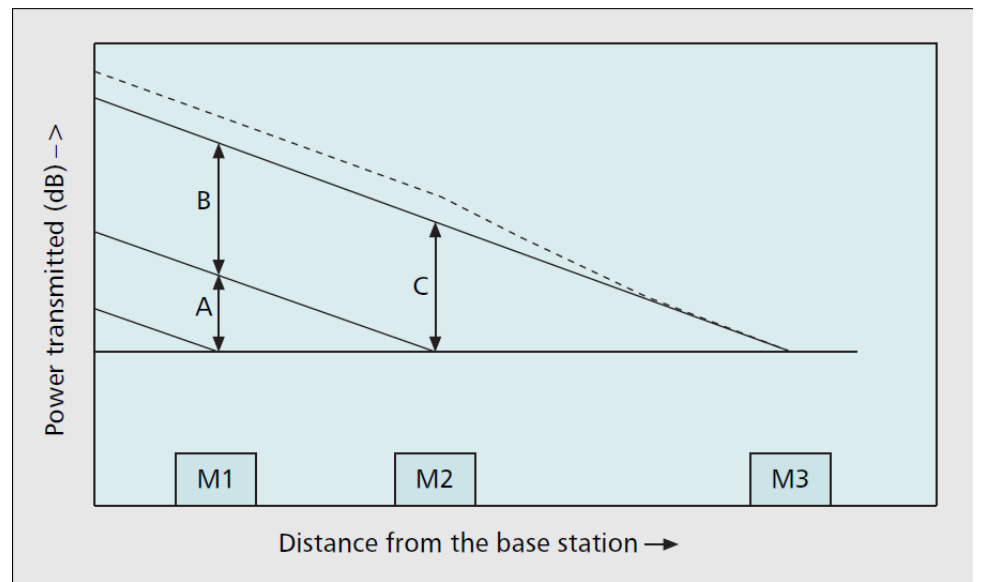

Figure 11. Superposition coding in cellular network [13]

The use of superposition coding in the context of SVC multicast transmission is as follows:

- Perform the list of SSs wishing to receive the video stream SVC.

- Establish respectively the different modulations of SSs in descending order of strength.

- List the flows of services related to multicast groups according to the size of the video layer that transports.

- Calculate the number of slots required for each physical flow of service with each modulation.

- Depending on the number of available physical slots, calculate for each modulation, the maximum flow of service beginning with the most important.

- Using the superposition coding by overlaying data from different modulations.

This algorithm is included in the admission control function at the BS, similar to the previous solution. To limit the number of superposed modulations, the BS should choose two or three modules most suitable to meet the maximum SSs of the cell.

Note that, unlike the multiple modulations mode, the redundancy is still present in the overlay technique. Indeed, the base layer is encoded for example as many times as there are modulations to superpose. This is due to the fact that SS can decode several modulations simultaneously. Despite this redundancy, the amount of resources used remains less than any other proposal.

In the next section, we evaluate by simulation the performance of the two solutions, we compare the results with the conventional method and we show the gain each time.

\section{Simulation Environment and results}

\subsection{Unicast Services support}

We consider the same topology in Figure 2., the link between BS1 and BS2 is assumed to be a reliable wired link since we focus essentially on WIMAX networks performance. We use QualNet Network Simulator [9] which implements PMP (point to multipoint) mode of IEEE802.16, and in which we implemented our XLO. We set IEEE 802.16 PHY parameters as mentioned in TABLE I.

We simulate a video streaming traffic from SS2 to SS4 with different scenarios using video traffic generator based on pre-encoded MPEG-4 traffic traces [8]. These traces provide three video qualities: high, medium and low video quality corresponding to three different data rate (see TABLE II. for more details). We developed a new scalable video streaming generator based on MPEG traces which is capable of stream switching by varying video quality and consequently varying video data rate.

In the next subsection, we describe four simulated case scenarios and discuss the simulation results. 
TABLE I. IEEE 802.16 PHY SIMULATION PARAMETERS

\begin{tabular}{|l|c|}
\hline Propagation channel frequency & $2.4 \mathrm{GHz}$ \\
\hline Channel bandwidth & $20 \mathrm{MHz}$ \\
\hline FFT size & 2048 \\
\hline Antenna gain & $12 \mathrm{~dB}$ \\
\hline Transmission Power & $20 \mathrm{~dB}$ \\
\hline Frame size & $20 \mathrm{~ms}$ \\
\hline
\end{tabular}

TABLE II. HIGH, MEDIUM AND LOW VIDEO QUALITY

\begin{tabular}{|c|c|c|c|}
\cline { 2 - 4 } \multicolumn{1}{c|}{} & \multicolumn{3}{c|}{ Video quality } \\
\cline { 2 - 4 } \multicolumn{1}{c|}{} & Medium & Low \\
\hline Frame rate & 25 frames $/ \mathrm{sec}$ & 25 frames $/ \mathrm{sec}$ & 25 frames $/ \mathrm{sec}$ \\
\hline Mean data rate & $766 \mathrm{Kbps}$ & $267 \mathrm{Kbps}$ & $153 \mathrm{Kbps}$ \\
\hline
\end{tabular}

\subsubsection{Scenario1: Normal conditions}

This first scenario evaluates the throughput of video streaming under normal condition, and assuming there are enough resources in the network. Figure 12. shows the simulation results for high, medium and low video quality. The obtained bit rate for each quality is indicated in the $\mathrm{Y}$ axis. These curves will serve as a baseline for better understanding the subsequent scenarios.

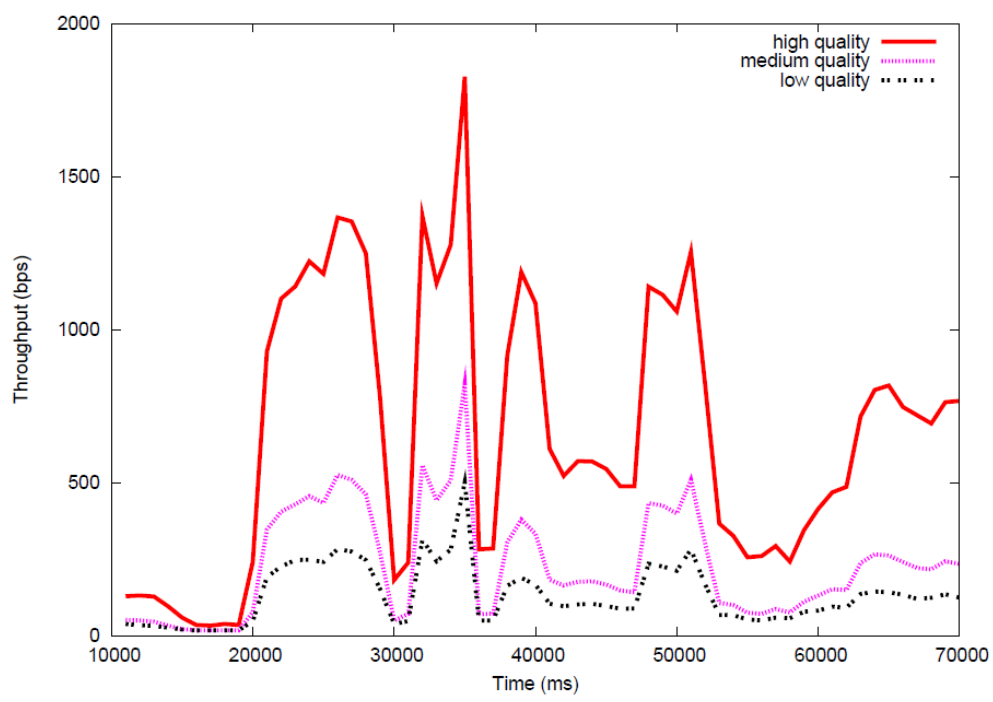

Figure 12. High, medium and low video quality data rate

\subsubsection{Scenario 2: Admission control adaptation}

In this scenario, we evaluate the performance of the proposed solution in the presence of an admission control mechanism. In addition to our scalable video traffic from SS2 to SS4, we added background traffic from SS1 and SS3 to SS5 with higher priority to disrupt video traffic. We choose real time CBR traffic with a high bit rate so that BS1 will not have enough resources to satisfy high video quality and force the video streaming server via the XLO to reduce its data rate until satisfaction as explained in Figure 4.

Background traffic runs during all simulation time, and video streaming traffic starts at $t=10 \mathrm{sec}$ for one minute long. TABLE III indicate background traffic data rate in each scenario. By default, the video streaming server starts transmitting with high video quality and keeps XLO adapting its data rate according to BS feedbacks.

In Figure 13. , we can observe how the application switches immediately from high to medium quality with only few packets belonging to high video quality, and then the curve takes the shape corresponding to the medium quality. The same behaviour is observed in Figure 14. where XLO moves the video quality down to a low level. In fact, CBR traffic data rate is high enough (30.75 Mbps) so that BS1 has no longer available resources to satisfy high or medium quality. 


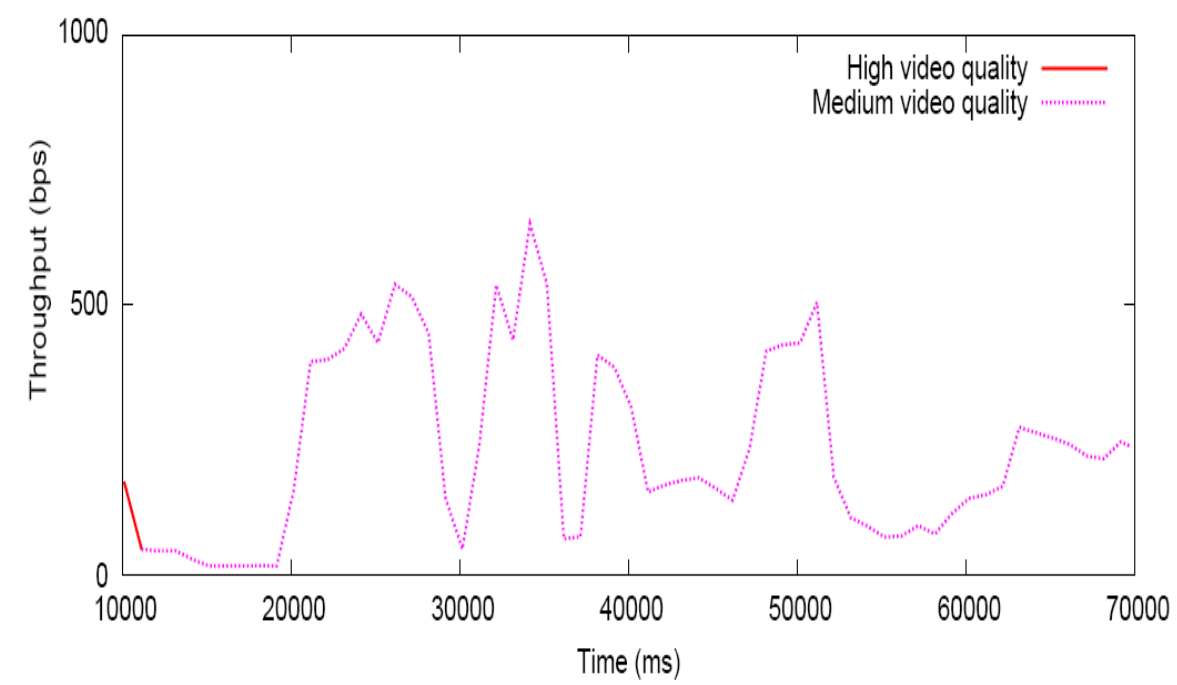

Figure 13 . High quality reduced to Medium quality video data rate in admission

TABLE III. SCENARIOS BACKGROUND TRAFFIC SETTINGS

\begin{tabular}{|c|l|c|l|}
\cline { 2 - 4 } \multicolumn{1}{c|}{} & \multicolumn{1}{c|}{ Description } & $\begin{array}{c}\text { Background } \\
\text { CBR traffic } \\
\text { data rate }\end{array}$ & \multicolumn{1}{c|}{$\begin{array}{c}\text { Related } \\
\text { figures }\end{array}$} \\
\hline \multirow{2}{*}{$\begin{array}{c}\text { Scenario } \\
2\end{array}$} & High reduced to medium quality in admission control & $30.6 \mathrm{Mbps}$ & Figure 13 \\
\cline { 2 - 5 } & High reduced to low quality in admission control & $30.75 \mathrm{Mbps}$ & Figure 14 \\
\hline $\begin{array}{c}\text { Scenario } \\
3\end{array}$ & High reduced to medium quality during lifetime streaming & $30.6 \mathrm{Mbps}$ & Figure 16 \\
\cline { 2 - 5 } & High reduced to low quality during lifetime streaming & $31 \mathrm{Mbps}$ & Figure 17 \\
\hline $\begin{array}{c}\text { Scenario } \\
4\end{array}$ & Low increased to High video quality during lifetime streaming & $\begin{array}{c}30.75 \mathrm{Mbps} \\
\text { Ends at } \\
40 \mathrm{sec}\end{array}$ & Figure 18 \\
\hline
\end{tabular}

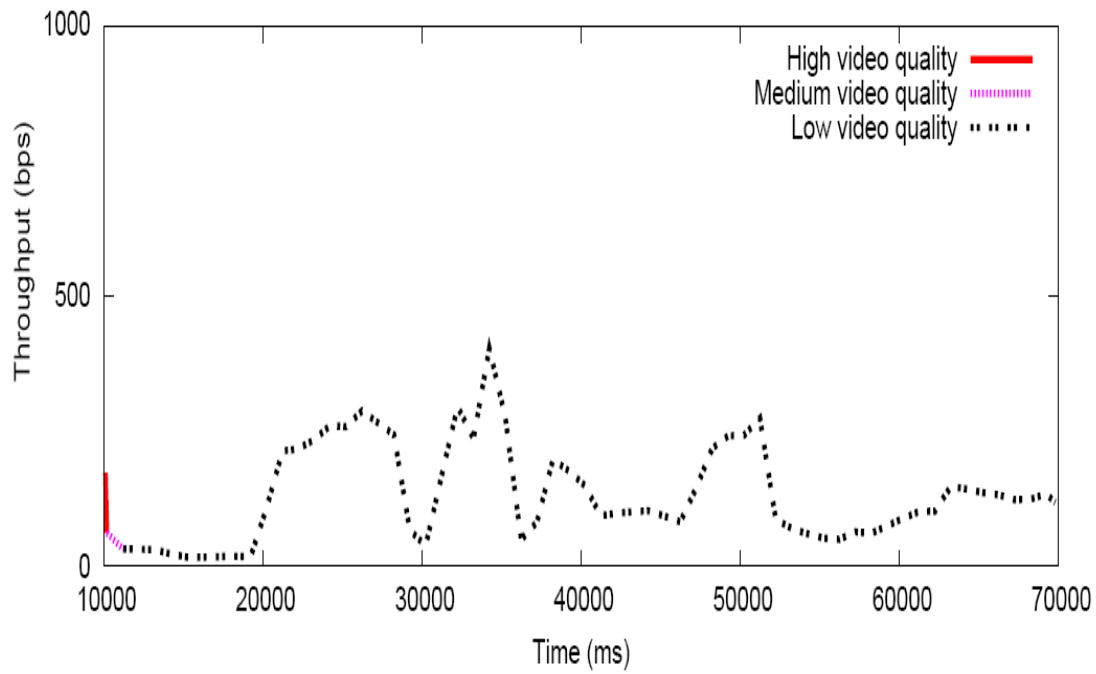

Figure 14. reduced to Low quality video data rate in admission

\subsubsection{Scenario 3: adaptation during video streaming (decrease video data rate)}

This scenario aims to show how XLO works during the life-time of the video streaming session; we simulate the same configuration of the precedent scenario. CBR data rate is chosen so as to give high video quality the chance to be accepted from the beginning of the streaming session. 
Then, at $\mathrm{t}=30 \mathrm{sec}$, we initiate another CBR traffic with higher priority. We manage its data rate so that BS will not have enough resources for already existing video traffic. The total data rate of background traffic is mentioned in TABLE III. We run this scenario without XLO, and we obtain the results given by Figure 15.

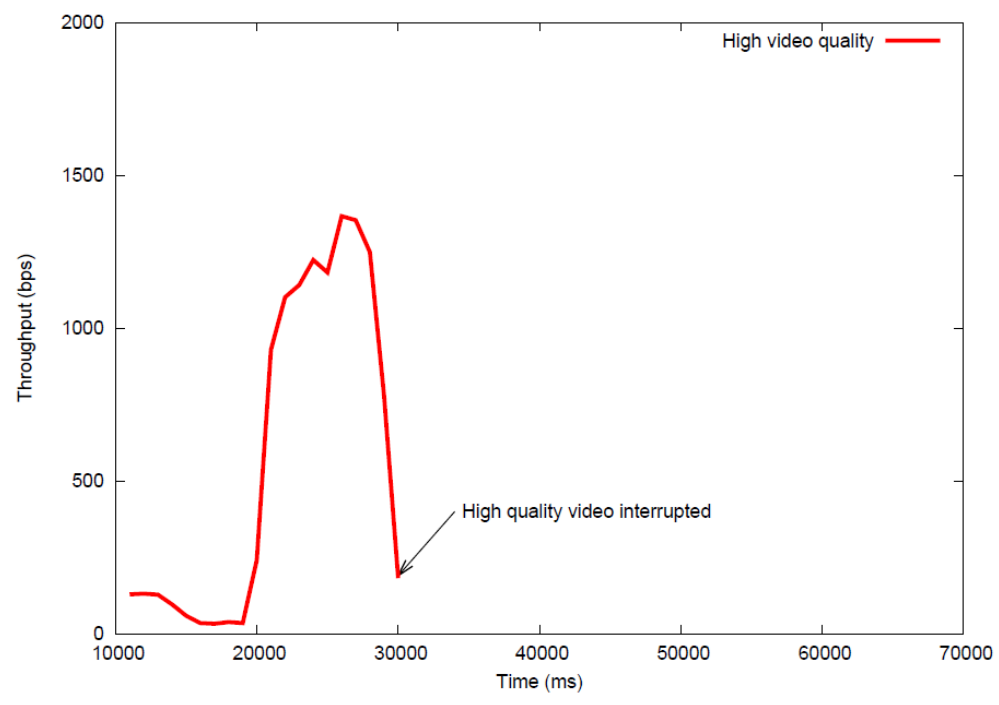

Figure 15. High quality video data rate interrupted during transmission (without XLO)

We can see that with no XLO, when BS cannot satisfy the high quality video data rate, a reject message is sent to SS2, the SF connection aborts and the video streaming traffic is simply interrupted while, with XLO, the results are different. In fact, thanks to our optimization in its scheduling algorithm, BS is aware of SFs related to scalable video streaming application. Consequently, as shown in Figure 5. , XLO will force the video streaming server-side application to adapt and reduce its data rate. Figure 16. shows the simulation results for a scenario where video data rate is reduced to the data rate of the medium video quality. Figure 17. shows simulation results for a scenario where video data rate is reduced multiple times until it reaches a low video quality.

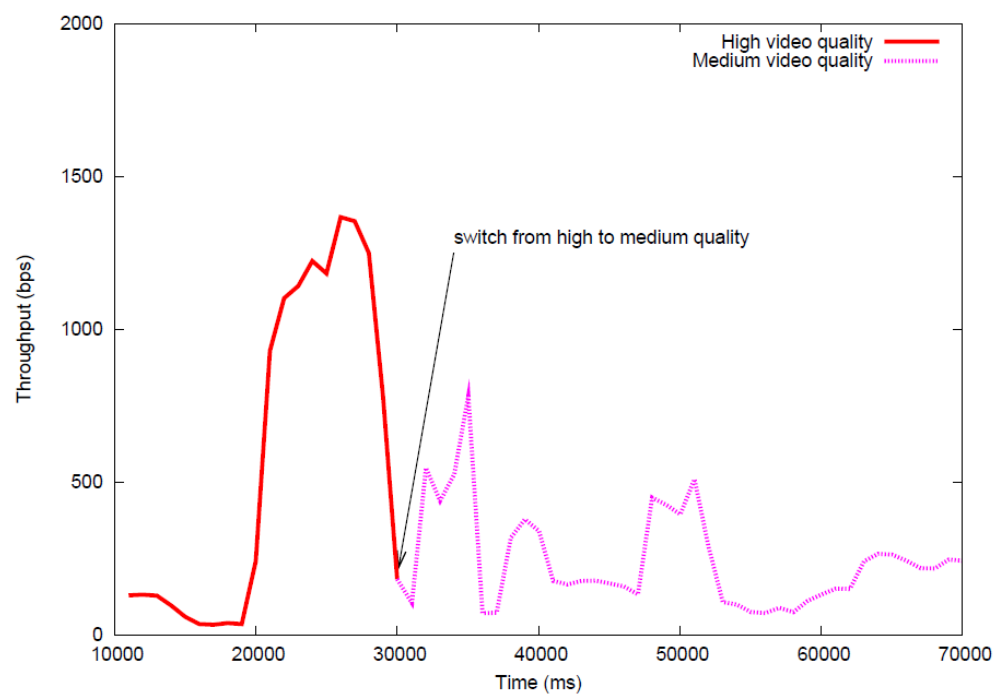

Figure 16. High reduced to Medium quality video data rate during transmission 


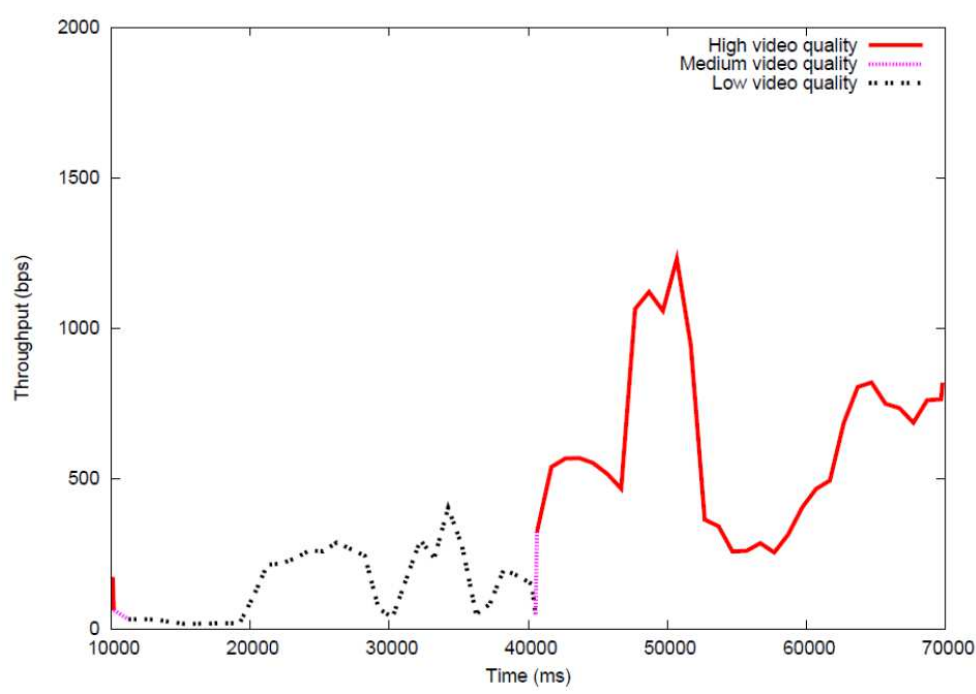

Figure 17. Low quality increased to High quality video data rate during transmission

\subsubsection{Scenario 4: adaptation during video streaming (increase video data rate)}

In this scenario, we reuse the same simulation settings as in scenario 2 with a background traffic of $30.75 \mathrm{Mbps}$ starting in the beginning of the simulation but ends at $t=40 \mathrm{sec}$ and not during all simulation time. As mentioned above, the video data rate will decrease, thanks to XLO admission control, until it reaches a low video quality. Then, at $\mathrm{t}=40 \mathrm{sec}$, background traffic stopped and the resources it used become available. When the XLO entity detects this availability, it informs the video streaming server in order to increase its video data rate. A first DSC request message is sent from SS to BS to reach medium video quality as mentioned in Figure 17. , and then a second DSC request is sent to reach high video quality.

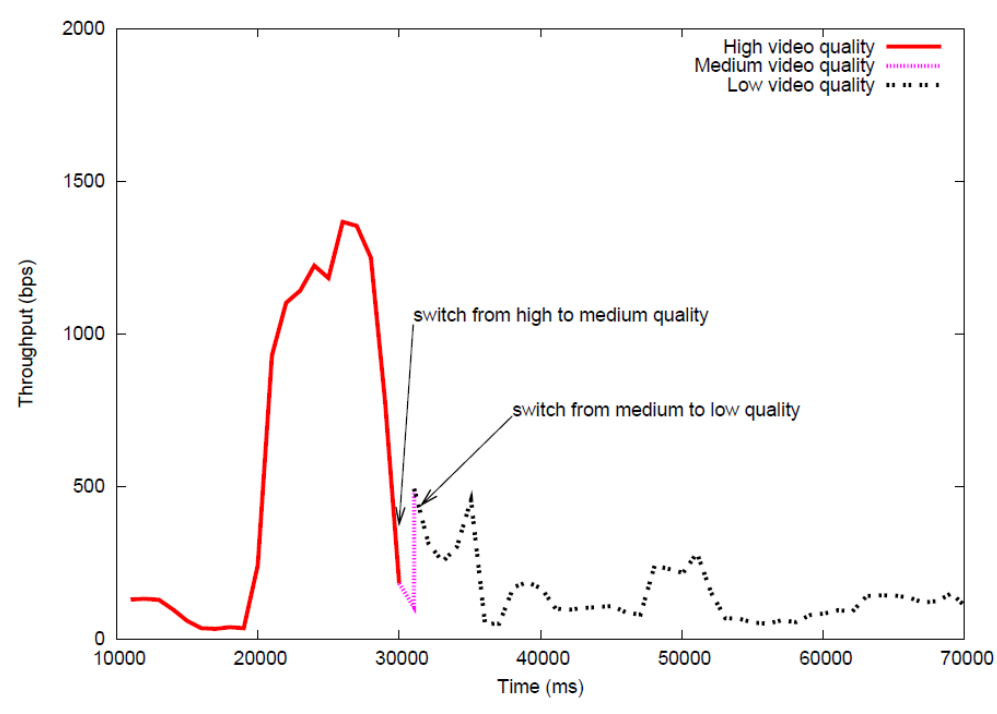

Figure 18. High reduced to Low quality video data rate during transmission

\subsection{Multicast Services Support}

We define in this subsection, 3 scenarios. First, we evaluate by simulation the classic case of SVC multicast transmission in WiMAX networks without any modification. Then, a first approach is implemented; it is to map multicast groups to different patterns of modulation. Finally, the third approach, we evaluate the SVC multicast transmission in a superposition coding.

\subsubsection{Simulation environment}

We used the QualNet simulator [9] to perform simulations of the various proposed mechanisms. The implementation is divided into two parts: 
- The SVC multicast server: We adapted a traffic generator QualNet already set to generate multiple streams, each corresponding to a video layer from a SVC traffic trace file. These traces represent the sizes of images, and the transmission time of each image. With the help of a program using the JSVM tool, we obtain log files from a real video coded using SVC. These traces contain in particular the size of images and their membership base layer or enhancement layers. The multicast stream is transmitted as an SVC video layer.

- Admission control: we have changed the resource allocation algorithms in the control of admission to the BS. New features are added to support SVC multicast video traffic. Two versions were implemented: one for multi-mode modulation that provides the best distribution between the modulations and multicast groups, and the second for the superposition coding to determine the number of video layers required for each modulation. For both mechanisms, the signalling between the BS and the SSs is provided by fields DL_MAP_IE MAP sent at the beginning of each frame.

The parameters of the IEEE 802.16 physical layer, common to all BSs of all simulations are provided in TABLE IV. The parameters of the SVC video used are provided in TABLE V. The 60-second video sequence is partitioned into several video layers. We note a base layer L0 and four enhancement layers L1, L2, L3 and L4.

TABLE IV. PHY LAYER SIMULATION PARAMETERS

\begin{tabular}{|l|c|}
\hline Propagation channel frequency & $2.4 \mathrm{GHz}$ \\
\hline Channel bandwidth & $20 \mathrm{MHz}$ \\
\hline FFT size & 2048 \\
\hline Antenna gain & $12 \mathrm{~dB}$ \\
\hline Transmission Power & $20 \mathrm{~dB}$ \\
\hline Frame size & $20 \mathrm{~ms}$ \\
\hline
\end{tabular}

TABLE V. SVC PARAMETERS

\begin{tabular}{|l|c|}
\hline Description & Football sequence \\
\hline Duration & 60 seconds \\
\hline Average Throughput & $\sim 160 \mathrm{Kbps}$ \\
\hline GOP Size & 16 images par GOP \\
\hline \multirow{2}{*}{ Number of Video Layers } & 5 (noted L0, .., L4) \\
\hline Average throughput for L0 & $\sim 37 \mathrm{Kbps}$ \\
L1 & $\sim 17 \mathrm{Kbps}$ \\
L2 & $\sim 28 \mathrm{Kbps}$ \\
L4 & $\sim 38 \mathrm{Kbps}$ \\
\end{tabular}

\subsubsection{Simulation results}

We perform a first set of simulation using a topology with three WIMAX cells connected through a wired network (Internet or LAN) to the SVC video server (Figure 19. ). A SS of each cell should receive the maximum video quality. In the first scenario, no changes at the BS are applied. The BS operates in classic mode as defined by default in the QualNet simulator.

We do not distinguish between SSs in terms of radio characteristics during scheduling. To do so, the 3 SSs are placed at the same distance from their BS, as shown inTABLE VI. . However, a difference exists between the SSs; traffic present in each cell is not the same. CBR traffic is added in the background to overload the cell. We modify the flow of traffic in each cell to observe its influence on the multicast video traffic SVC. 

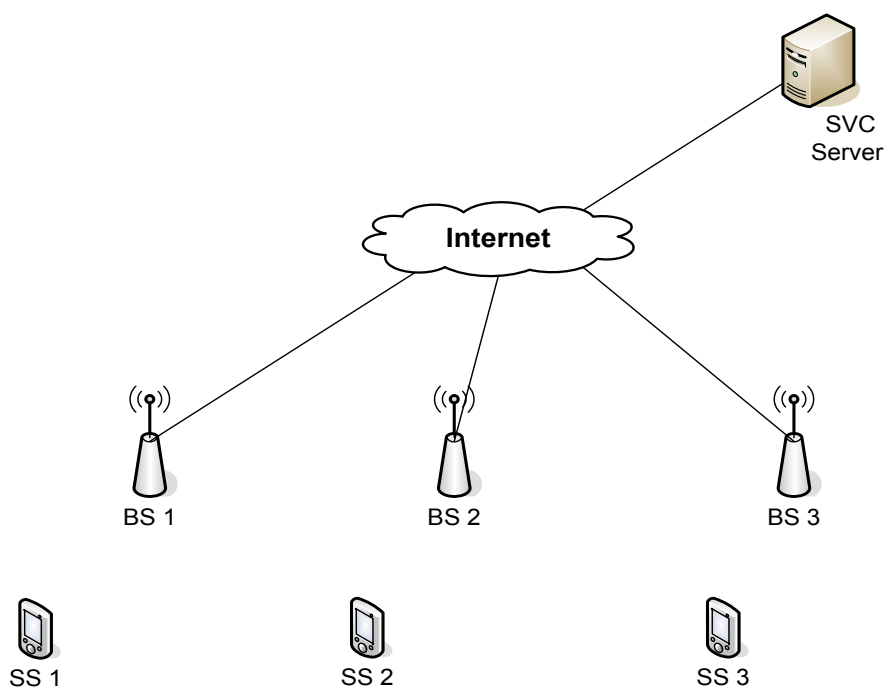

Figure 19. Multiple cells topology

TABLE VI. SCENARIO: IDENTICAL SS IN DIFFERENT CELLS

\begin{tabular}{|c|c|c|c|}
\cline { 2 - 4 } \multicolumn{1}{c|}{} & SS 1 & SS 2 & SS 3 \\
\hline $\begin{array}{c}\text { Distance From the BS } \\
(\mathrm{m})\end{array}$ & 350 & 350 & 350 \\
\hline CBR Flow (Mbps) & 19.97 & 19.90 & 0 \\
\hline
\end{tabular}

Note that the CBR flow is added with a higher priority than SVC flow, the goal is to limit bandwidth in each cell to force SS to select a lower video quality. The results of this simulation are illustrated in Figure 20. .

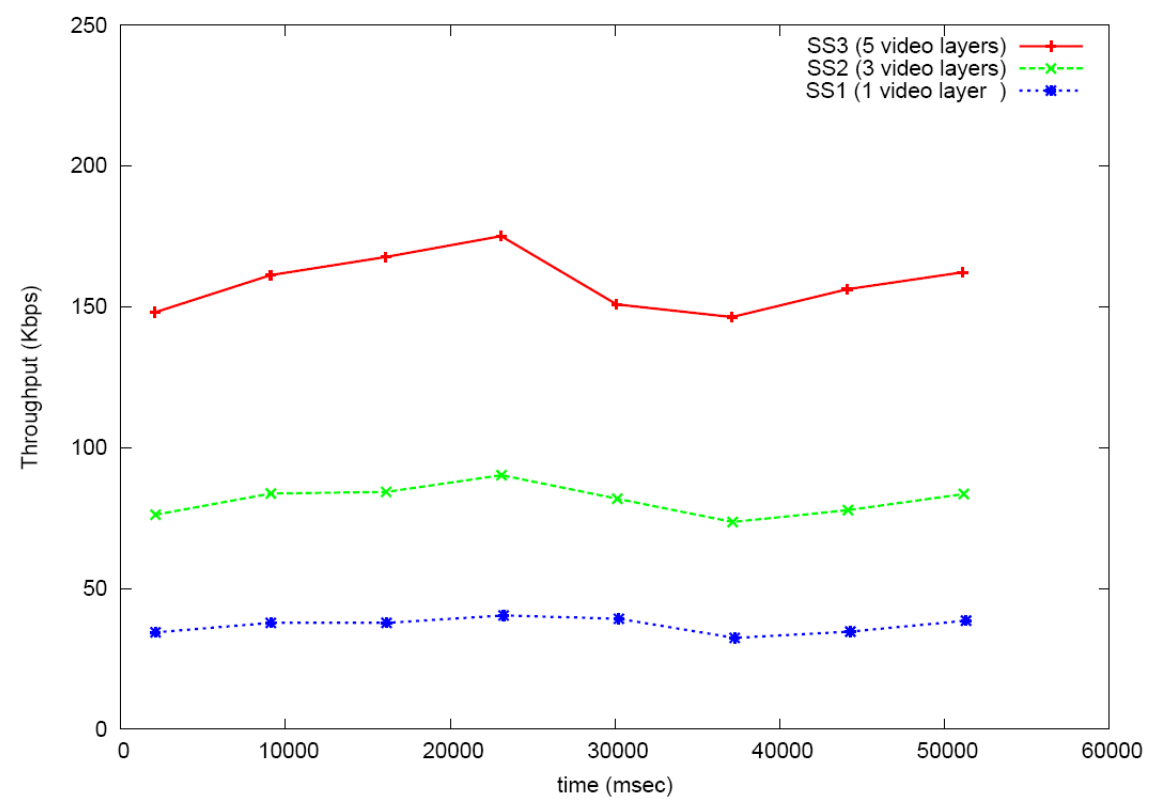

Figure 20. Scenario: Identical SS in different cells

We note that the three SSs do not receive the same quality video. SS1 has the lowest quality by receiving the base layer L0 only, all resources being allocated; the BS was unable to allocate more bandwidth to SS1. The highest video quality is perceived by SS3 which received the 5 layers of video (L0 to L4), with no traffic disturbance, all the resources of the BS were available to SS3. Finally, SS2 was entitled to a quality medium containing the base layer L0 and two enhancement layers L1 and L2. This simulation allows us to validate the usefulness of the decomposition of SVC flow into several multicast groups. Each SS, depending on the available bandwidth acquires a different quality video. In addition, multicast groups, containing layers of unnecessary improvements, are not transmitted to the BS, their flow stops at the last router in common with other BS. 
The rest of the simulations described in this section are made with the topology illustrated in Figure 21 . It includes a single cell WiMAX SSs and 3 placed at different distances from the BS. Distance of SSs is chosen so that the modulations SSs assigned to either different from each other as shown in TABLE VII.

TABLE VII. SCENARIO: DIFFERENT SS IN THE SAME CELL

\begin{tabular}{|c|c|c|c|}
\cline { 2 - 4 } \multicolumn{1}{c|}{} & SS 1 & SS 2 & SS 3 \\
\hline Distance from the BS (m) & 100 & 350 & 580 \\
\hline CBR flow (Mbps) & 19.80 & 19.80 & 19.80 \\
\hline Modulation / Coding Rate & 64QAM & 16QAM & QPSK \\
\hline
\end{tabular}
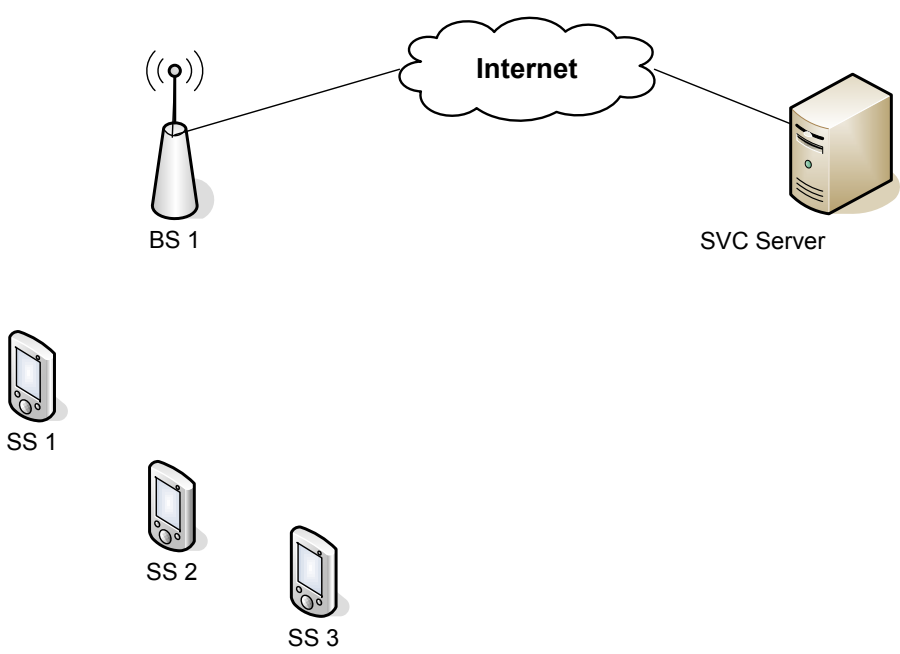

Figure 21. Scenario: Different SS in the same cells

Three scenarios are defined. First, we conduct a simulation in the simple modulation mode without any modification. Then we simulate the multi-mode modulation and finally, we finish by simulating the superposition coding mode. In all three simulations, we keep the same parameters of 802.16 physical layer; and the same simulation parameters as the distance of their SSs to BS. In addition, a background CBR traffic with a high priority is introduced in all simulations. In order to compare accurately the different proposals, we assume in each simulation scenario, that the BS has the same radio resources to serve the present SSs.

\subsubsection{Simple coding scheme}

In the simple coding mode, each SS receives the data with the modulation assigned by the BS. In order to serve all the present SSs, it is necessary that the layers L0, 1, 2, 3 and 4 are coded with the three modulations (QPSK, 16 QAM, 64 QAM). For this, the BS must allocate the necessary resources for 15 burst data. Precisely, we chose the flow of CBR traffic (19.8 million bps) to prevent this from happening. Indeed, the BS is unable to satisfy all the SSs. The results of this simulation are depicted in Figure 22.

We note that none of SSs could reach the optimal video quality. Indeed, SS1 reached an average rate of about 80 Kbps, which corresponds to the average flow layers L0, L1 and L2 combined. SS2 and SS3 received L0 and L1. By analyzing the log files of this simulation, we could see that the resources available to the BS were used as follows:

- The L0 layer coded QPSK for SS3, in 16QAM and 64QAM for SS2 to SS1,

- The L1 layer coded QPSK for SS3, in 16QAM and 64QAM for SS2 to SS1,

- The layer L2 coded 64QAM for SS1, 


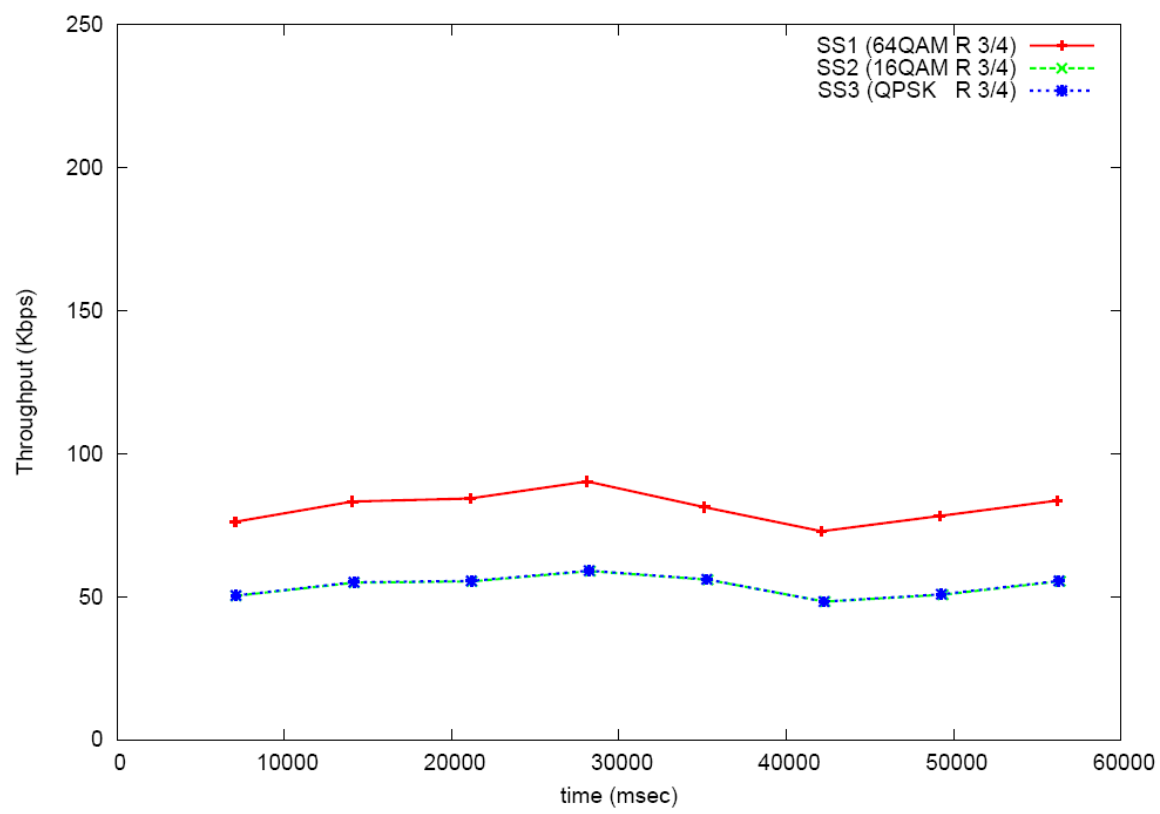

Figure 22. Scenario: Simple coding scheme

Through these simulation results, we find that the multicast transmission has not been beneficial for the three heterogeneous SSs since there were redundant layers L0 and L1. In the next scenario, we make changes to optimize use of available resources.

\subsubsection{Multiple coding scheme}

In this scenario, we perform the simulation with the same parameters as in the previous scenario. The results are illustrated in Figure 23. With the optimized use of modulations, the SSs are able to improve the quality of their videos. Indeed, SS3 reached a rate equivalent to 3 video layers instead of only two layers in the classic mode. SS2 acquires two additional enhancement layers SS1 and manages to have the maximum video quality. The minimum video quality in this scenario is the best video quality achieved in the classic mode, this represents a considerable gain.

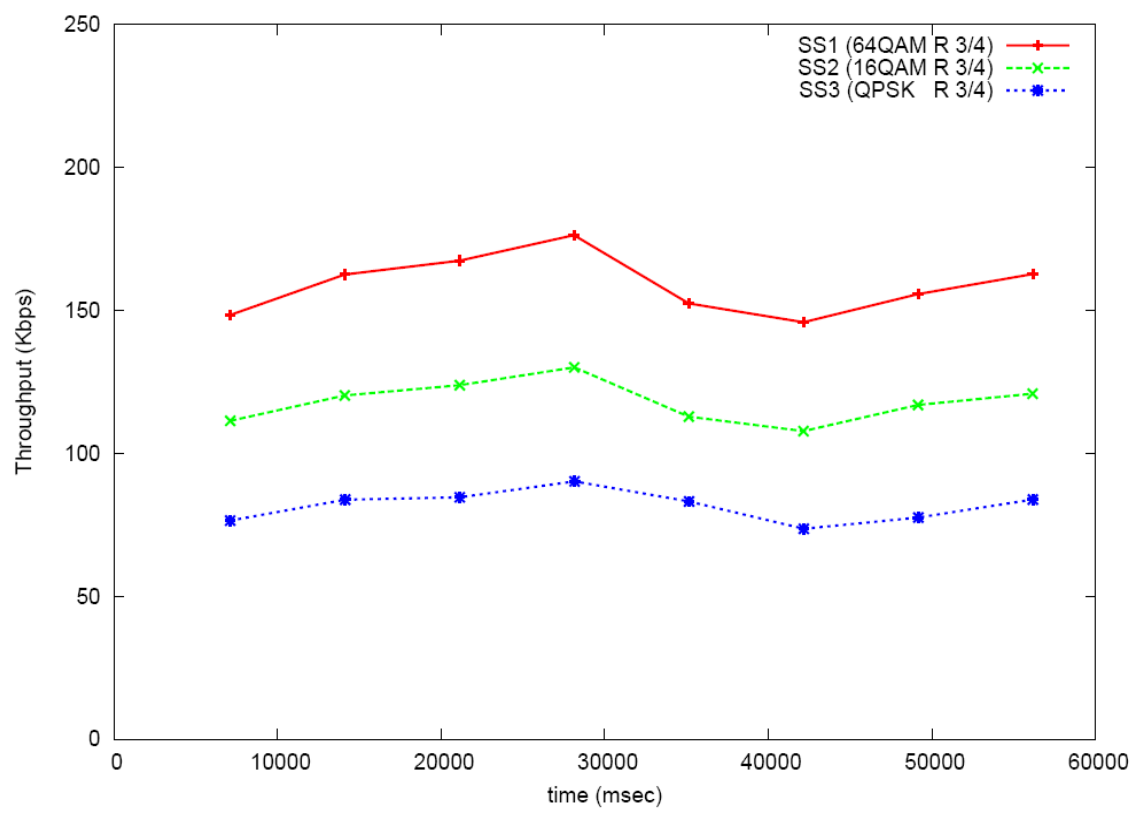

Figure 23. Scenario: Mulitple coding scheme

The simulation traces on the physical layer of the BS provide us with the following details: the layer $\mathrm{L} 0, \mathrm{~L} 1$ and L2 were coded QPSK, 16QAM, L3 and L4 in 64QAM. Thus, three burst with three modulations are inserted into the frame. Therefore, each SS is able to decode the burst with modulation assigned by default, and the burst 
with a more robust modulation as the modulation by default. It is the role of the BS to inform the SSs about the burst availability.

In addition, no redundancy is introduced; each video layer is encoded once and with a single modulation. Resources became available, compared to the simple coding mode, will allow the addition of other video layers and thus improve the video quality in each SS

\subsubsection{Superposition coding scheme}

In this scenario, we use the same parameters as in other scenarios. The simulation results about the superposed coding scheme are provided in Figure 24. We notice a significant improvement. The average flow of the video stream observed in average on each cell is significantly higher than in other scenarios. Indeed, SS1 and SS2 reach the maximum quality of the video (all layers), the SS3 receives 4 video layers (only a single enhancement layer is missing).

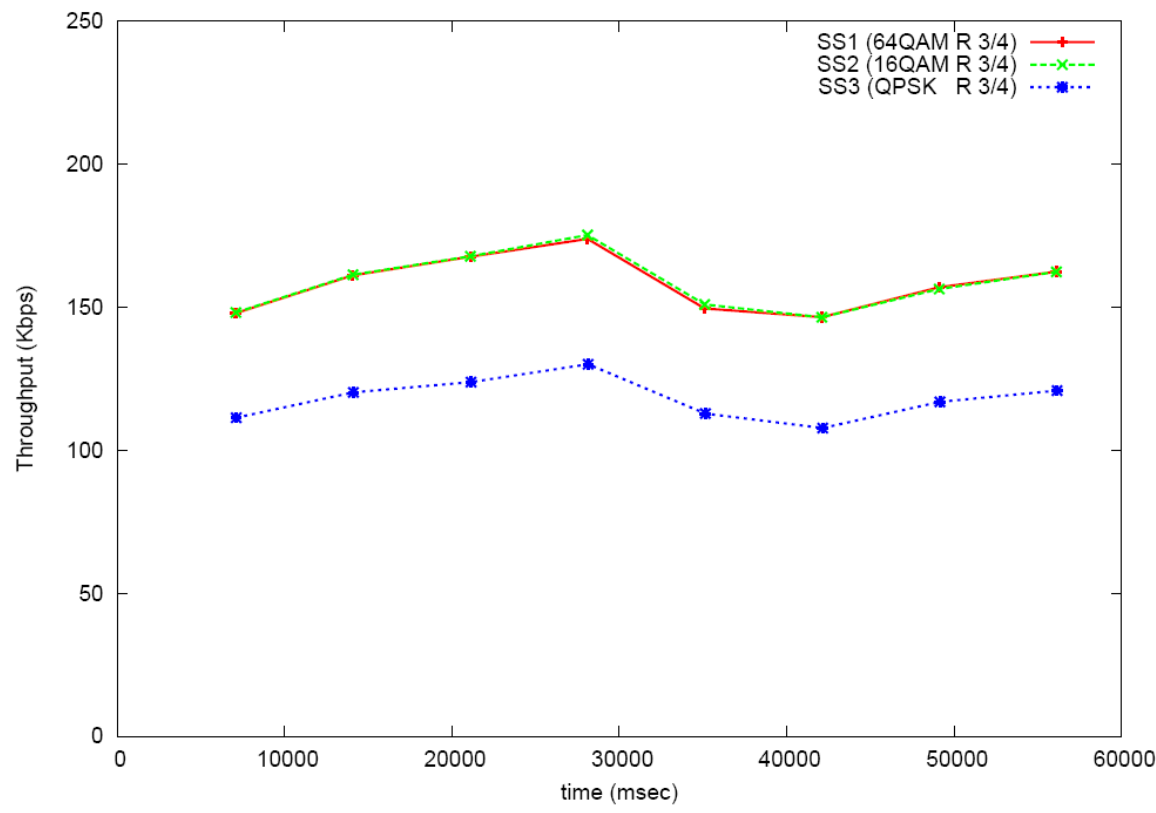

Figure 24. Scenario: Superposition coding scheme

At the physical layer level, the resource was allocated as follow: for each SS, the BS allocates a burst of data encoded with the default modulation used by the SS. Three burst in total are allocated, the size of each burst must not exceed the number of the available slots in the physical frame independently of the modulation used. In our simulation, it is clear that the number of physical slots available is smaller than the number of slots required for a burst that contains all the video layers and encoded with QPSK. For this reason, SS3 received four of the five layers. For the burst encoded in 16QAM and 64QAM, it was possible to transport the 5 layers.

We summarize the results of three scenarios in TABLE VIII. The table shows the number of video layers received by each SS in every scenario. We note that the conventional method fails to satisfy any SS with a maximum video quality while the BS has allocated all the available resources. The multiple modulations mode provides a considerable gain and the optimized use of modulation schemes for each SS brings fruit. This mode offers the best SS quality observed compared to the classic mode. Finally, the superposition coding represents the maximum optimization of radio resources compared to all scenarios.

TABLE VIII. NUMBER OF VIDEO LAYER RECEIVED

\begin{tabular}{|c|c|c|c|}
\cline { 2 - 4 } \multicolumn{1}{c|}{} & SS1 & SS2 & SS3 \\
\hline Superposition Coding & 5 & 5 & 4 \\
\hline Multiple Modulations & 5 & 4 & 3 \\
\hline Simple Modulation & 3 & 2 & 2 \\
\hline
\end{tabular}

The two methods we presented are suitable for the disadvantageous SSs, i.e., those which are the most distanced from the BS. Indeed, we note that SS2 and SS3 are stations that were benefited most from this optimization as the both proposals provide them a quality approaching the maximum. 


\section{Conclusion}

In this paper, we introduced a cross layer solution for adaptive video streaming applications in IEEE 802.16 networks for both unicast and multicast traffic. For the unicast traffic we proposed XLO which collects information provided by the MAC layer, essentially SF management messages exchanged between BS and SS and assigns new optimised parameters to video streaming server-side application. The ultimate goal is to adapt video data rate according to resource availability at the MAC layer. The conducted simulations showed that adaptation can be performed during the admission control and the life-time of video streaming sessions and allows a continuous video stream with no interruption especially if there are no available resources for the high video quality. Our future work includes the extension of the current optimizer to multi hop relay WIMAX networks. Moreover, the cross layer optimization described in this paper follows a bottom-up approach where application benefits from lower layer parameters. It will be interesting to complement it with a top-down approach to make the MAC layer adaptable according to video application requirements.

In the multicast case, we have proposed several mechanisms for transmitting multicast in WiMAX networks. The problem studied is to find the best compromise between, on one hand, the diversity of client stations in terms of bandwidth, resources and radio conditions, and in the other hand, the hierarchical structure of SVC video coding system suitable for heterogeneous receivers. We studied the operation of such application in a typical WiMAX network, we identified problems and we have proposed solutions to address them.

Two techniques were introduced. First, the intelligent use of different modulations at the SSs allowed a significant gain in resources. This technique highlights the compatibility of some stations with more robust modulations in relation to modulation by default assigned by the BS. The redundancy of certain video layers is then omitted. Then, coding using multiple modulations allows optimal use of available resources in the cell. The principle of this technique lies in the fact that the radio bearer, to send data to a station far from the BS, the same time allows the integration of data sent to a nearby station.

Optimization of resource utilization remains a hot topic. The mechanisms described in this paper are adapted to the IEEE 802.16 OFDMA physical layer. In fact, the decomposition of the frame into several OFDM symbols or physical slots allows multiple users to access the channel and offers, therefore, easier manipulation algorithms for scheduling and resource allocation.

\section{Reference}

[1] IEEE Std 802.16, "IEEE Standard for Local and metropolitan area networks. Part 16: Air Interface for Fixed Broadband Wireless Access Systems", October 2004.

[2] IEEE Std 802.16e, "IEEE Standard for Local and metropolitan area networks Part 16: Air Interface for Fixed and Mobile Broadband Wireless Access Systems. Amendment 2: Physical and Medium Access Control Layers for Combined Fixed and Mobile Operation in Licensed Bands and Corrigendum 1", February 2006.

[3] Yi-Ting Mai; Chun-Chuan Yang; Yu-Hsuan Lin, "Cross-Layer QoS Framework in the IEEE 802.16 Network," Advanced Communication Technology, The 9th International Conference on , vol.3, no., pp.2090-2095, 12-14 Feb. 2007

[4] Y. T. Mai, C. C. Yang, and Y. H. Lin, "Design of the Cross-Layer QoS Framework for the IEEE 802.16 PMP Networks," IEICE Transactions on Communications, vol. E91-B, no. 5, pp. 1360-1369, May 2008.

[5] Noordin, K.A.; Markarian, G., "Cross-Layer Optimization Architecture for WiMAX Systems," Personal, Indoor and Mobile Radio Communications, 2007. PIMRC 2007. IEEE 18th International Symposium on , vol., no., pp.1-4, 3-7 Sept. 2007

[6] D.-K. Triantafyllopoulou, N. Passas, and A. Kaloxylos, "A Cross-Layer Optimization Mechanism for Multimedia Traffic over IEEE 802.16 Networks", European Wireless 2007, Paris, France, Apr. 2007.

[7] Fan Li, G. Liu, L. He, "Application-driven cross-layer design of multiuser H.264 video transmission over wireless networks", Proceedings of the 2009 International Conference on Wireless Communications and Mobile Computing: Connecting the World Wirelessly, pp. 176-180, 21-24 Juin 2009.

[8] MPEG-4 and H.263 Video Traces for Network Performance Evaluation.[Online].Available:http://www.tkn.tuberlin.de/research/trace/trace.html

[9] Scalable Network Technologies (SNT). QualNet. .[Online]. Available: http://www.qualnet.com/

[10] Ch. Neumann and V. Roca, Scalable Video Streaming over ALC (SVSoA): a Solution for the Large Scale Multicast Distribution of Videos, Research Report, INRIA Rh.one-Alpes, Plan_ete project, March 2003.

[11] M. Luby, et. al., “Asynchronous Layered Coding (ALC) Protocol Instantiation”, IETF Internet RFC 3450, December 2002.

[12] J. She, X. Yu, P. Ho, and E. Yang. A cross-layer design framework for robust IPTV services over IEEE 802.16 networks. IEEE JSAC, 27(2):235-245, Feb. 2009.

[13] J. She, Xiang Yu, Fen Hou, Pin-Han Ho, and En-Hui Yang, ”A Framework of Cross-Layer Superposition Coded Multicast for Robust IPTV Services over WiMAX,” IEEE Wireless Communications and Networking Conference, 2008., pp. 3139-3144, 2008

[14] J. She et al., "IPTV over WiMAX: Key Success Factors, Challenges, and Solutions", IEEE Communications Magazine, vol. 45, no 8, Aug. 2007, pp.87-93 